Document downloaded from:

http://hdl.handle.net/10251/99862

This paper must be cited as:

Albanese, A.; Bonet Solves, JA.; Ricker, WJ. (2015). Spectrum and compactness of the Cesàro operator on weighted I_p spaces. Journal of the Australian Mathematical Society. 99(3):287-314. doi:10.1017/S1446788715000221

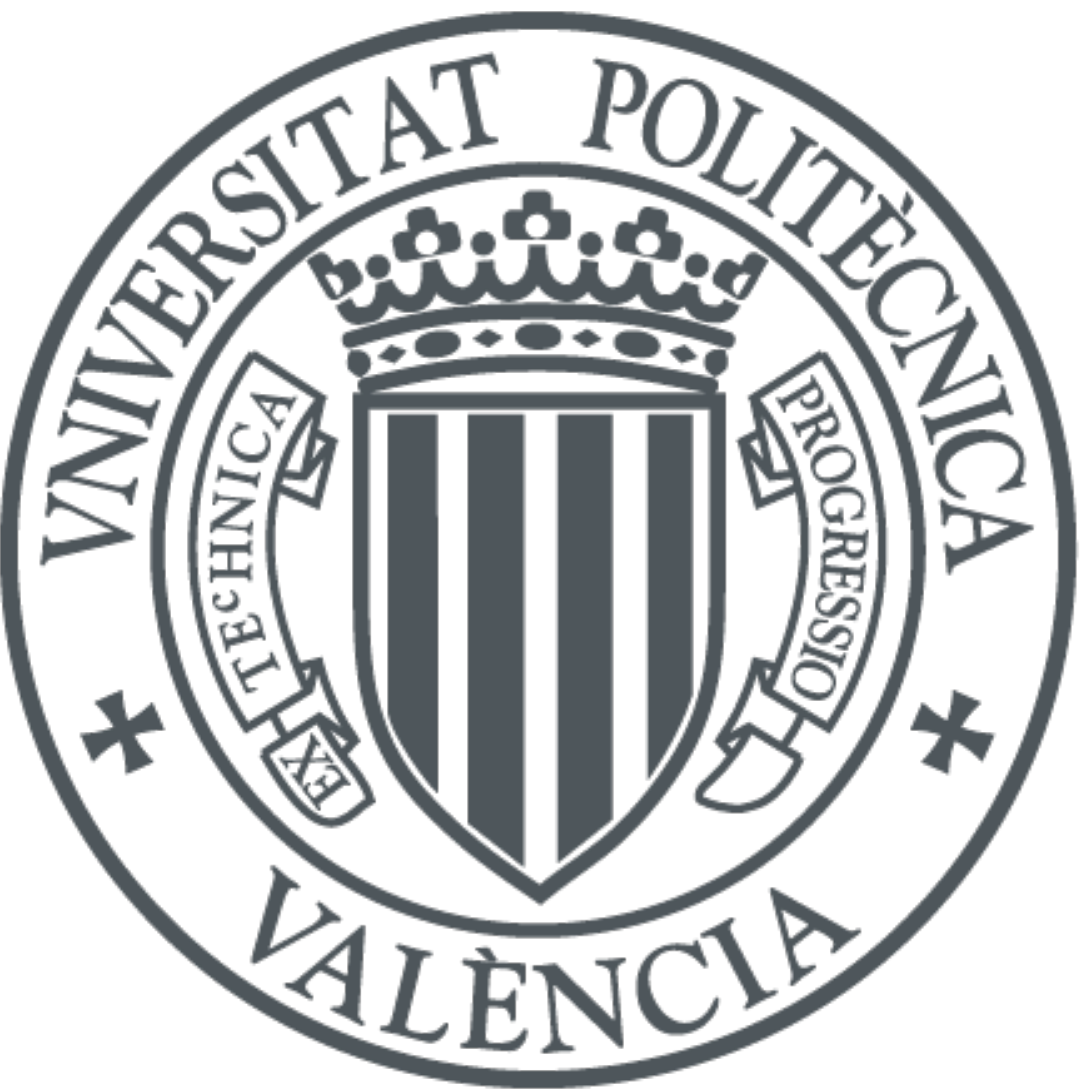

The final publication is available at

http://doi.org/10.1017/S1446788715000221

Copyright Cambridge University Press

Additional Information 


\title{
SPECTRUM AND COMPACTNESS OF THE CESÀRO OPERATOR ON WEIGHTED $\ell_{P}$ SPACES
}

\author{
ANGELA A. ALBANESE, JOSÉ BONET and WERNER J. RICKER
}

(May 4, 2015)

\begin{abstract}
An investigation is made of the continuity, the compactness and the spectrum of the Cesàro operator $\mathrm{C}$ when acting on the weighted Banach sequence spaces $\ell_{p}(w), 1<p<\infty$, for a positive, decreasing weight $w$, thereby extending known results for $C$ when acting on the classical spaces $\ell_{p}$. New features arise in the weighted setting (e.g., existence of eigenvalues, compactness) which are not present in $\ell_{p}$.
\end{abstract}

Keywords and phrases: Cesàro operator, weighted $\ell_{p}$ space, spectrum, compact operator.

\section{Introduction}

The discrete Cesàro operator $C$ is defined on the linear space $\mathbb{C}^{\mathbb{N}}$ (consisting of all scalar sequences) by

$$
\mathrm{C} x:=\left(x_{1}, \frac{x_{1}+x_{2}}{2}, \ldots, \frac{x_{1}+\ldots+x_{n}}{n}, \ldots\right), \quad x=\left(x_{n}\right)_{n \in \mathbb{N}} \in \mathbb{C}^{\mathbb{N}} .
$$

The operator $C$ is said to act in a vector subspace $X \subseteq \mathbb{C}^{\mathbb{N}}$ if it maps $X$ into itself. Of particular interest is the situation when $X$ is a Banach space. The fundamental questions in this case are: Is C: $X \rightarrow X$ continuous and, if so, what is the spectrum of C: $X \rightarrow X$ ? Amongst the classical Banach spaces $X \subseteq \mathbb{C}^{\mathbb{N}}$ where precise answers are known we mention $\ell_{p}$ $(1<p<\infty)$, [6], [15], and $c_{0}$, [15], [19], and both $c, \ell_{\infty}$, [1], [15], as well as $\operatorname{ces}_{p}, p \in\{0\} \cup(1, \infty)$, [8], the Bachelis spaces $N^{p}, 2 \leq p<\infty$, [9], and the spaces of bounded variation $b v_{0},[18]$, and bounded $p$-variation $b v_{p}$, $1 \leq p<\infty$, [2]. In all of these cases, the operator norm of C : $X \rightarrow X$ equals

Mathematics Subject Classification 2010: Primary 47A10, 47B37; Secondary 46B45, 47A16, 47A30.

(c) XXXX Australian Mathematical Society $0263-6115 / \mathrm{XX} \$ \mathrm{~A} 2.00+0.00$ 
its spectral radius and $C$ has at most one eigenvalue, namely 1 . There is no claim that this list of spaces (and references) is complete.

The aim of this paper is to investigate the two questions mentioned above for $\mathrm{C}$ acting on the weighted Banach spaces $\ell_{p}(w)$. To be precise, let $w=$ $(w(n))_{n=1}^{\infty}$ be a bounded sequence, always assumed to be strictly positive. Define the space

$$
\ell_{p}(w):=\left\{x=\left(x_{n}\right)_{n \in N} \in \mathbb{C}^{\mathbb{N}}:\|x\|_{p, w}:=\left(\sum_{n=1}^{\infty}\left|x_{n}\right|^{p} w(n)\right)^{1 / p}<\infty\right\}
$$

for each $1<p<\infty$, equipped with the norm $\|\cdot\|_{p, w}$. Observe that $\ell_{p}(w)$ is isometrically isomorphic to $\ell_{p}$ via the linear multiplication operator

$$
\Phi_{w}: \ell_{p}(w) \rightarrow \ell_{p}, \quad x=\left(x_{n}\right)_{n \in \mathbb{N}} \rightarrow \Phi_{w}(x):=\left(w(n)^{1 / p} x_{n}\right)_{n \in \mathbb{N}} .
$$

Therefore, each $\ell_{p}(w)$ is a Banach space. The dual space $\left(\ell_{p}(w)\right)^{\prime}$ of $\ell_{p}(w)$ is the Banach space $\ell_{p^{\prime}}(v)$, where $\frac{1}{p}+\frac{1}{p^{\prime}}=1$ (i.e., $p^{\prime}$ is the conjugate exponent of $p$ ) and $v(n)=w(n)^{-p^{\prime} / p}$ for $n \in \mathbb{N}$. In particular, $\ell_{p}(w)$ is reflexive and separable for $1<p<\infty$. Moreover, the canonical unit vectors $e_{k}:=$ $\left(\delta_{k n}\right)_{n \in \mathbb{N}}$, for $k \in \mathbb{N}$, form an unconditional basis in $\ell_{p}(w)$ for $1<p<\infty$. If $\inf _{n \in \mathbb{N}} w(n)>0$, then $\ell_{p}(w)=\ell_{p}$ with equivalent norms and we are in the standard situation. Accordingly, we are mainly interested in the case when $\inf _{n \in \mathbb{N}} w(n)=0$.

By Hardy's inequality, [14, Theorem 326, p.239], for every $1<p<\infty$ the restriction of the Cesàro operator $C: \mathbb{C}^{\mathbb{N}} \rightarrow \mathbb{C}^{\mathbb{N}}$ as given in (1) defines a bounded linear operator from $\ell_{p}$ into itself with operator norm equal to $p^{\prime}$. Denote these operators by $\mathrm{C}^{(p)}$ so that $\left\|\mathrm{C}^{(p)}\right\|=p^{\prime}$. In Section 2, where the papers [5], [11], [12] are relevant, we discuss various aspects of the continuity of $\mathrm{C}$ when it is restricted to $\ell_{p}(w), 1<p<\infty$; denote this operator by $\mathrm{C}^{(p, w)}$ whenever it is continuous.

For any Banach space $X$, let $I$ denote the identity operator on $X$ and $\mathcal{L}(X)$ denote the space of all continuous linear operators from $X$ into itself. The spectrum and the resolvent set of $T \in \mathcal{L}(X)$ are denoted by $\sigma(T)$ and $\rho(T)$, respectively; see [10, Ch. VII], for example. The set of all eigenvalues of $T$, called the point spectrum of $T$, is denoted by $\sigma_{p t}(T)$. The spectral radius $r(T):=\sup \{|\lambda|: \lambda \in \sigma(T)\}$ of $T$ always satisfies $r(T) \leq\|T\|,[10$, p.567].

Section 3 is devoted to an analysis of the spectrum of $\mathrm{C}$ when acting in $\ell_{p}(w)$. The main result is Theorem 3.3; it is complemented by Examples 3 which clarify the scope of this theorem. Unlike for $\mathrm{C}^{(p)}$, it can happen that $\sigma_{p t}\left(\mathrm{C}^{(p, w)}\right) \neq \emptyset$. Actually, $\mathrm{C}^{(p, w)}$ can even have infinitely many eigenvalues; see Proposition 3.4. The final section deals with the compactness of $\mathrm{C}^{(p, w)}$. Relevant is how fast $w$ decreases to 0; see Proposition 4.1, Theorem 4.2, Corollary 4.3 and Proposition 4.4. Unlike for C acting in the classical Banach 
spaces mentioned in the opening paragraph, it may happen in $\ell_{p}(w)$ that $r\left(C^{(p, w)}\right)<\left\|C^{(p, w)}\right\| ;$ see Proposition 4.1.

\section{Continuity of $C$ in weighted $\ell_{p}$ spaces}

Some of the concepts and results from [12] that are quoted in this section actually have their origins in the paper [11]. We begin with the following fact.

Lemma 2.1. Let $w=(w(n))_{n=1}^{\infty}$ be a positive sequence and $1<p<\infty$. Then the Cesàro operator $\mathrm{C}$ maps $\ell_{p}(w)$ continuously into itself if, and only if,

$$
\sup _{m \in \mathbb{N}}\left(\sum_{k=1}^{m} w(k)^{-p^{\prime} / p}\right)^{-1}\left(\sum_{n=1}^{m} \frac{w(n)}{n^{p}}\left(\sum_{k=1}^{n} w(k)^{-p^{\prime} / p}\right)^{p}\right)<\infty,
$$

i.e., if, and only if, there exists $K>0$ such that

$$
\sum_{n=1}^{m} \frac{w(n)}{n^{p}}\left(\sum_{k=1}^{n} w(k)^{-p^{\prime} / p}\right)^{p} \leq K\left(\sum_{k=1}^{m} w(k)^{-p^{\prime} / p}\right), \quad m \in \mathbb{N} .
$$

Moreover, if the constant $K$ satisfying (2) is chosen as small as possible, then the operator norm of $\mathrm{C}$ is at most $p^{\prime} K^{1 / p}$.

Proof. Let $T_{w}: \mathbb{C}^{\mathbb{N}} \rightarrow \mathbb{C}^{\mathbb{N}}$ denote the linear operator defined by

$$
T_{w} x:=\left(\frac{w(n)^{1 / p}}{n} \sum_{k=1}^{n} w(k)^{-1 / p} x_{k}\right)_{n \in \mathbb{N}}, \quad x=\left(x_{n}\right)_{n \in \mathbb{N}} \in \mathbb{C}^{\mathbb{N}} .
$$

Then $\Phi_{w} C=T_{w} \Phi_{w}$. Since $\Phi_{w}$ is isometric from $\ell_{p}(w)$ onto $\ell_{p}$, it follows that $C$ maps $\ell_{p}(w)$ continuously into itself if, and only if, $T_{w}$ maps $\ell_{p}$ continuously into itself. But, the matrix of $T_{w}$ is factorable (cf. $[5, \S 4]$ with $a_{n}=w(n)^{1 / p} / n$ and $b_{k}=w(k)^{-1 / p}$ for $\left.1 \leq k \leq n\right)$ and so it follows from [5, Theorem 2] that $T_{w} \in \mathcal{L}\left(\ell^{p}\right)$ if, and only if, (2) holds.

The proof of Theorem 2 in [5] yields that the operator norm of $C$ is at most $p^{\prime} K^{1 / p}$.

Proposition 2.2. Let $w=(w(n))_{n=1}^{\infty}$ be a decreasing, positive sequence and $1<p<\infty$. Then the Cesàro operator $\mathrm{C}^{(p, w)} \in \mathcal{L}\left(\ell_{p}(w)\right)$ and satisfies

$$
1<\left(\frac{1}{w(1)} \sum_{n=1}^{\infty} \frac{w(n)}{n^{p}}\right)^{1 / p} \leq\left\|\mathrm{C}^{(p, w)}\right\| \leq p^{\prime} .
$$


Proof. Fix $m \in \mathbb{N}$. Because $w$ is decreasing, we have

$$
\begin{aligned}
& \sum_{n=1}^{m} \frac{w(n)}{n^{p}}\left(\sum_{k=1}^{n} w(k)^{-p^{\prime} / p}\right)^{p}=\sum_{n=1}^{m}\left(\frac{w(n)^{1 / p}}{n} \sum_{k=1}^{n} w(k)^{-p^{\prime} / p}\right)^{p} \\
& \leq \sum_{n=1}^{m}\left(\frac{w(n)^{1 / p}}{n} \cdot \frac{n}{w(n)^{p^{\prime} / p}}\right)^{p}=\sum_{n=1}^{m} w(n)^{-p^{\prime} / p}
\end{aligned}
$$

which is precisely (2) with $K=1$. So, Lemma 2.1 implies that $\mathrm{C}$ is continuous on $\ell_{p}(w)$ with $\left\|\mathrm{C}^{(p, w)}\right\| \leq p^{\prime}$.

For an alternate proof of the continuity of $\mathrm{C}^{(p, w)}$, based directly on Hardy's inequality in $\ell_{p}$, see [12, Proposition 5.1].

Since $T_{w}=\Phi_{w} C^{(p, w)} \Phi_{w}^{-1}$, with $\Phi_{w}$ mapping the closed unit ball of $\ell_{p}(w)$ onto that of $\ell_{p}$ and $\Phi_{w}^{-1}$ mapping the closed unit ball of $\ell_{p}$ onto that of $\ell_{p}(w)$, it follows that $\left\|T_{w}\right\|=\left\|\mathrm{C}^{(p, w)}\right\|$. Of course,

$$
\Phi_{w}^{-1} x=\left(w(n)^{-1 / p} x_{n}\right)_{n \in \mathbb{N}}, \quad x \in \ell_{p} .
$$

Substituting $x=e_{1}$ into (3) it follows that

$$
\left\|\mathrm{C}^{(p, w)}\right\|=\left\|T_{w}\right\| \geq\left\|T_{w} e_{1}\right\|_{p}=\left(\frac{1}{w(1)} \sum_{n=1}^{\infty} \frac{w(n)}{n^{p}}\right)^{1 / p} \geq\left(1+\frac{w(2)}{w(1) 2^{p}}\right)^{1 / p}>1 .
$$

See also [12, Proposition 5.5].

Some comments regarding Proposition 2.2 are in order. As noted above, for each $1<p<\infty$ we have $\left\|\mathrm{C}^{(p)}\right\|=p^{\prime}$ and, for a positive, decreasing weight $w$, that (4) holds. These estimates are not the best possible in general. Denote by $\delta_{p}(w)$ the set of all decreasing, non-negative sequences in $\ell_{p}(w)$ and define

$$
\Delta_{p, w}\left(\mathrm{C}^{(p, w)}\right):=\sup \left\{\left\|\mathrm{C}^{(p, w)} x\right\|_{p, w}: x \in \delta_{p}(w),\|x\|_{p, w}=1\right\} \leq\left\|\mathrm{C}^{(p, w)}\right\| .
$$

The following result follows from Propositions $6.3,6.5$ and 6.6 of [12].

Proposition 2.3. Let $1<p<\infty$ and $w(n)=1 / n^{\alpha}, n \in \mathbb{N}$, for a fixed $\alpha>0$. Then

$$
\max \left\{m_{1}, m_{2}\right\} \leq \Delta_{p, w}\left(C^{(p, w)}\right) \leq\left\|C^{(p, w)}\right\| \leq M_{2}(r):=[r \zeta(r+\alpha)]^{r / p},
$$

for $1 \leq r \leq p$, where $m_{1}:=p /(p+\alpha-1)$ and $m_{2}:=\zeta(p+\alpha)^{1 / p}$, with $\zeta$ denoting the Riemann zeta function. Moreover, for $\alpha \leq r<(p+\alpha)$, it is also the case that

$$
\left\|C^{(p, w)}\right\| \leq M_{3}(r):=\left(\frac{p}{p+\alpha-r}\right)^{1 / p^{\prime}} \zeta\left(1+\frac{r}{p^{\prime}}+\frac{\alpha}{p}\right)^{1 / p} .
$$


We provide some relevant examples.

Example 1. (i) For $w(n)=1 / n^{\alpha}$, if $\alpha=0.9$ and $p=1.1$, then $\max \left\{m_{1}, m_{2}\right\} \simeq 1.572$ and $M_{2}(1)=M_{3}(0.9) \simeq 1.663$ (see pp.15-16 of [12]) and so Proposition 2.3 shows that

$$
1.572 \leq\left\|\mathrm{C}^{(p, w)}\right\| \leq 1.663 .
$$

On the other hand, $p^{\prime}=11$ and so Proposition 2.2 only yields $\left\|C^{(p, w)}\right\| \leq 11$.

(ii) Still for $w(n)=1 / n^{\alpha}$, but now with $\alpha=0.5$ and $p=2$, we have $m_{1}=4 / 3$ and $M_{3}(3 / 4) \simeq 1.593$ (see p.16 of [12]) so that Proposition 2.3 reveals that

$$
\frac{4}{3} \leq\left\|C^{(p, w)}\right\| \leq 1.593 .
$$

In this case, $p^{\prime}=2$ and so Proposition 2.2 only yields $\left\|\mathrm{C}^{(p, w)}\right\| \leq 2$.

(iii) Again for $w(n)=1 / n^{\alpha}$, with $\alpha>0$, it follows (in the notation of Proposition 2.3) that

$$
\left(\frac{1}{w(1)} \sum_{n=1}^{\infty} \frac{w(n)}{n^{p}}\right)^{1 / p}=\left(\sum_{n=1}^{\infty} \frac{1}{n^{p+\alpha}}\right)^{1 / p}=\zeta(p+\alpha)^{1 / p}=m_{2} .
$$

Hence, the lower bound in (4) reduces to $m_{2} \leq\left\|\mathrm{C}^{(p, w)}\right\|$ whereas (5) yields $\max \left\{m_{1}, m_{2}\right\} \leq\left\|\mathrm{C}^{(p, w)}\right\|$. Of course, (4) applies to more general weights $w$.

The following example is not a consequence of Proposition 2.3.

Example 2. Let $p=2$ and set $w(n)=2^{-n}$ for $n \in \mathbb{N}$. The proof of Proposition 2.2 yields that $\left\|\mathrm{C}^{(2, w)}\right\|=\left\|T_{w}\right\|$. Recall, via (3), that

$$
T_{w} x=\left(\frac{1}{n 2^{n / 2}} \sum_{k=1}^{n} 2^{k / 2} x_{k}\right)_{n \in \mathbb{N}}, \quad x=\left(x_{n}\right)_{n \in \mathbb{N}} \in \ell_{2} .
$$

For every $x \in \ell_{2}$, it follows via the Cauchy-Schwarz inequality and the identity $\sum_{k=1}^{n} r^{k}=\left(r-r^{n+1}\right) /(1-r)$, for $r \neq 1$, that

$$
\begin{aligned}
\left\|T_{w} x\right\|_{2}^{2} & =\sum_{n=1}^{\infty} \frac{1}{n^{2} 2^{n}}\left|\sum_{k=1}^{n} 2^{k / 2} x_{k}\right|^{2} \leq \sum_{n=1}^{\infty} \frac{1}{n^{2} 2^{n}}\left(\sum_{k=1}^{n} 2^{k}\right)\left(\sum_{k=1}^{n}\left|x_{k}\right|^{2}\right) \\
& \leq\|x\|_{2}^{2} \sum_{n=1}^{\infty} \frac{1}{n^{2} 2^{n}}\left(2^{n+1}-2\right)=\|x\|_{2}^{2} \sum_{n=1}^{\infty} \frac{2\left(1-2^{-n}\right)}{n^{2}} .
\end{aligned}
$$

Accordingly, $\left\|T_{w}\right\| \leq\left(\sum_{n=1}^{\infty} \frac{2\left(1-2^{-n}\right)}{n^{2}}\right)^{1 / 2}$. Observe that

$$
\sum_{n=1}^{\infty} \frac{\left(1-2^{-n}\right)}{n^{2}}=\frac{\pi^{2}}{6}-\int_{0}^{1 / 2} \frac{-\log (1-t)}{t} d t
$$


because of the fact that $\frac{\pi^{2}}{6}=\sum_{n=1}^{\infty} \frac{1}{n^{2}}$ and the identity

$$
\int_{0}^{1 / 2} \frac{-\log (1-t)}{t} d t=\int_{0}^{1 / 2} \sum_{n=0}^{\infty} \frac{t^{n}}{(n+1)}=\sum_{n=1}^{\infty} \frac{1}{n^{2} 2^{n}} .
$$

The function $f(t)=\frac{-\log (1-t)}{t}$ for $t \in(0,1]$, with $f(0):=1$, is positive, continuous and increasing on $[0,1)$ and so

$$
1=f(0) \leq f(t) \leq f\left(\frac{1}{2}\right)=2 \log 2, \quad t \in[0,1 / 2],
$$

which implies that $-\log 2 \leq-\int_{0}^{1 / 2} \frac{-\log (1-t)}{t} d t \leq-\frac{1}{2}$. Consequently,

$$
\sum_{n=1}^{\infty} \frac{2\left(1-2^{-n}\right)}{n^{2}} \leq 2\left(\frac{\pi^{2}}{6}-\frac{1}{2}\right) \simeq 2.2898
$$

and so

$$
\left\|C^{(2, w)}\right\|=\left\|T_{w}\right\| \leq \sqrt{\left(\frac{\pi^{2}}{3}-1\right)} \simeq 1.513<p^{\prime}=2 .
$$

Direct calculation yields

$$
\left\|T_{w} e_{1}\right\|_{2}=\left(2 \sum_{n=1}^{\infty} \frac{1}{n^{2} 2^{n}}\right)^{1 / 2} \geq\left(2 \sum_{n=1}^{3} \frac{1}{n^{2} 2^{n}}\right)^{1 / 2} \simeq 1.073
$$

and so we have

$$
1.073 \leq\left\|\mathrm{C}^{(2, w)}\right\| \leq \sqrt{\left(\frac{\pi^{2}}{3}-1\right)} \simeq 1.513
$$

see also Proposition 2.2.

\section{Spectrum of $C^{(p, w)}$}

The aim of this section is to provide some detailed knowledge of the spectrum of $\mathrm{C}^{(p, w)}$. Unlike for the classical Cesàro operators $\mathrm{C}^{(p)} \in \mathcal{L}\left(\ell_{p}\right)$, for $1<p<\infty$, it can now happen that eigenvalues appear.

Given a (strictly) positive, bounded sequence $w=(w(n))_{n \in \mathbb{N}}$ and $1<$ $p<\infty$, let $S_{w}(p):=\left\{s \in \mathbb{R}: \sum_{n=1}^{\infty} \frac{1}{n^{s} w(n)^{p^{1} / p}}<\infty\right\}$. In case $S_{w}(p) \neq \emptyset$ we define $s_{p}:=\inf S_{w}(p)$. Note that $\frac{p^{\prime}}{p}=\frac{1}{p-1}$, for every $1<p<\infty$. Moreover, let $R_{w}:=\left\{t \in \mathbb{R}: \sum_{n=1}^{\infty} n^{t} w(n)<\infty\right\}$. In case $R_{w} \neq \mathbb{R}$ we define $t_{0}:=\sup R_{w}$. 
Fix $1<p<\infty$ and let $w(n)=2^{-n p / p^{\prime}}$ for $n \in \mathbb{N}$. Then $S_{w}(p)=\emptyset$, i.e., it can happen that $S_{w}(p)$ is empty. However, in the event that $S_{w}(p) \neq \emptyset$, then $s_{p} \geq 1$. Indeed, for any fixed $s \in \mathbb{R}$ we have

$$
\sum_{n=1}^{\infty} \frac{1}{n^{s} w(n)^{p^{\prime} / p}} \geq\|w\|_{\infty}^{-p^{\prime} / p} \sum_{n=1}^{\infty} \frac{1}{n^{s}} .
$$

So, whenever $s \in S_{w}(p)$ it follows that $\sum_{n=1}^{\infty} \frac{1}{n^{s}}<\infty$, that is, $s>1$. Hence, $S_{w}(p) \subseteq(1, \infty)$ which implies that $s_{p} \geq 1$. Moreover, for any $r>s \in S_{w}(p)$ we have

$$
\sum_{n=1}^{\infty} \frac{1}{n^{r} w(n)^{p^{\prime} / p}}<\sum_{n=1}^{\infty} \frac{1}{n^{s} w(n)^{p^{\prime} / p}}
$$

and so also $r \in S_{w}(p)$. Accordingly, whenever $S_{w}(p) \neq \emptyset$, then it is an infinite interval, i.e., $S_{w}(p)=\left[s_{p}, \infty\right)$ or $S_{w}(p)=\left(s_{p}, \infty\right)$ with $s_{p} \geq 1$. It is a consequence of $(7)$ that $1 \notin S_{w}(p)$, for all $1<p<\infty$ and all positive, bounded sequences $w$.

In the event that $a_{w}:=\inf _{n \in \mathbb{N}} w(n)>0$ it follows that necessarily $s_{p}=$ 1. Indeed, in this case $w(n)^{-p^{\prime} / p} \leq a_{w}^{-p^{\prime} / p}, n \in \mathbb{N}$, which implies that $\frac{1}{n^{s} w(n)^{p^{\prime} / p}} \leq \frac{a_{w}^{-p^{\prime} / p}}{n^{s}}$, for all $n \in \mathbb{N}$ and $s \in \mathbb{R}$. Hence, $(1, \infty) \subseteq S_{w}(p)$ and so $s_{p} \leq 1$. Since we are assuming that $S_{w}(p) \neq \emptyset$, we already know that $s_{p} \geq 1$. Accordingly, $s_{p}=1$.

Let $1<p<\infty$ and fix $\alpha>0$. For $w(n)=1 / n^{\alpha p / p^{\prime}}$ and any $s \in \mathbb{R}$ it follows that $\sum_{n=1}^{\infty} \frac{1}{n^{s} w(n)^{p^{\prime} / p}}=\sum_{n=1}^{\infty} \frac{1}{n^{s-\alpha}}<\infty$ precisely when $s>(1+\alpha)$ and so $s_{p}=1+\alpha$. Hence, given any $\beta>1$ and $1<p<\infty$, there exists a positive, decreasing weight $w \downarrow 0$ such that $S_{w}(p)=(\beta, \infty)$, i.e., $s_{p}=\beta$.

Concerning the set $R_{w}$, a similar discussion applies. For $w(n)=2^{-n}$ it turns out that $R_{w}=\mathbb{R}$ with $t_{0}=\infty$. However, if $R_{w} \neq \mathbb{R}$, then $t_{0}$ is finite with $t_{0} \geq-1$ and $R_{w}=\left(-\infty, t_{0}\right)$ or $R_{w}=\left(-\infty, t_{0}\right]$. Moreover, $R_{w}=\emptyset$ is not possible as $\sum_{n=1}^{\infty} n^{t} w(n) \leq\|w\|_{\infty} \sum_{n=1}^{\infty} n^{t}<\infty$ whenever $t<-1$. If $a_{w}>0$, then necessarily $t_{0}=-1$ but, $-1 \notin R_{w}$ as $\sum_{n=1}^{\infty} n^{t} w(n) \geq a_{w} \sum_{n=1}^{\infty} n^{t}$ for all $t \in \mathbb{R}$.

The following result clarifies the connection between $s_{p}$ and $t_{0}$.

Proposition 3.1. Let $w=(w(n))_{n \in \mathbb{N}}$ be a bounded, strictly positive sequence.

(i) For each $1<p<\infty$ such that $S_{w}(p) \neq \emptyset$ we have

$$
t_{0} \leq \frac{s_{p} p}{p^{\prime}}=(p-1) s_{p}
$$

In particular, $R_{w} \neq \mathbb{R}$ whenever there exists $p \in(1, \infty)$ with $S_{w}(p) \neq \emptyset$.

(ii) If $R_{w} \neq \mathbb{R}$, then $S_{w}(p) \subseteq\left[1+\frac{t_{0}}{(p-1)}, \infty\right)$, for every $1<p<\infty$. 
(iii) Suppose that $1<p<\infty$ satisfies $S_{w}(p) \neq \emptyset$. Then

$$
S_{w}(p) \subseteq S_{w}(q), \quad q \in[p, \infty) .
$$

In particular, $S_{w}(q) \neq \emptyset$ and $s_{q} \leq s_{p}$ whenever $q \geq p$.

(iv) If $S_{w}(p)=\emptyset$ for some $1<p<\infty$, then $S_{w}(q)=\emptyset$ for all $1<q \leq p$.

Proof. (i) Suppose that $S_{w}(p) \neq \emptyset$. Fix $s>s_{p}$. Since $\sum_{n=1}^{\infty} \frac{1}{n^{s} w(n)^{p^{\prime} / p}}<$ $\infty$, there exists $N \in \mathbb{N}$ such that $\frac{1}{n^{s} w(n)^{p^{\prime} / p}} \leq 1$ for $n \geq N$ and hence, $n^{s p / p^{\prime}} w(n) \geq 1$ for $n \geq N$. So, the series $\sum_{n=1}^{\infty} n^{s p / p^{\prime}} w(n)$ diverges which yields that $t_{0} \leq \frac{s p}{p^{\prime}}$. Accordingly, $t_{0} \leq \frac{s_{p} p}{p^{\prime}}$. In particular, $R_{w} \neq \mathbb{R}$.

(ii) Fix $p \in(1, \infty)$ and any $t<t_{0}$, in which case $\sum_{n=1}^{\infty} n^{t} w(n)<\infty$. Hence, there exists $K \in \mathbb{N}$ such that $n^{t} \leq \frac{1}{w(n)}$ for $n \geq K$, that is, $n^{t p^{\prime} / p} \leq$ $\frac{1}{w(n)^{p^{\prime} / p}}$ for $n \geq K$. So, for any $s \in \mathbb{R}$ we have (as $\frac{1}{n^{s}}>0$ for $n \in \mathbb{N}$ ) that

$$
\frac{1}{n^{s-\left(t p^{\prime} / p\right)}}=\frac{n^{t p^{\prime} / p}}{n^{s}} \leq \frac{1}{n^{s} w(n)^{p^{\prime} / p}}, \quad n \geq K
$$

Choose now $s \leq 1+\frac{t p^{\prime}}{p}$. It follows from the previous inequality that $\sum_{n=1}^{\infty} \frac{1}{n^{s} w(n)^{p^{\prime} / p}}$ diverges. Hence, $\sum_{n=1}^{\infty} \frac{1}{n^{s} w(n)^{p^{\prime} / p}}$ diverges whenever $s \leq$ $1+\frac{t p^{\prime}}{p}$, for some $t<t_{0}$, that is, whenever $s \in\left(-\infty, 1+\frac{t_{0} p^{\prime}}{p}\right)$. So, $S_{w}(p) \subseteq$ $\left[1+\frac{t_{0} p^{\prime}}{p}, \infty\right)=\left[1+\frac{t_{0}}{(p-1)}, \infty\right)$. have

(iii) Fix $s \in S_{w}(p)$, i.e., $\sum_{n=1}^{\infty} \frac{1}{n^{s} w(n)^{p^{\prime} / p}}<\infty$. For every $1<q<\infty$ we

$$
\sum_{n=1}^{\infty} \frac{1}{n^{s} w(n)^{q^{\prime} / q}}=\sum_{n=1}^{\infty} \frac{1}{n^{s} w(n)^{p^{\prime} / p}} \cdot w(n)^{\frac{p^{\prime}}{p}-\frac{q^{\prime}}{q}} \leq\|w\|_{\infty}^{\frac{p^{\prime}}{p}-\frac{q^{\prime}}{q}} \sum_{n=1}^{\infty} \frac{1}{n^{s} w(n)^{p^{\prime} / p}},
$$

which is finite provided that $\frac{p^{\prime}}{p} \geq \frac{q^{\prime}}{q}$. This is equivalent to $\left(p^{\prime}-1\right) \geq\left(q^{\prime}-1\right)$, that is, to $q \geq p$. Hence, whenever $q \geq p$ we have $S_{w}(p) \subseteq S_{w}(q)$ which clearly implies $S_{w}(q) \neq \emptyset$ and $s_{q} \leq s_{p}$.

(iv) Follows immediately from part (iii).

Define $\Sigma:=\left\{\frac{1}{n}: n \in \mathbb{N}\right\}$ and let $\Sigma_{0}:=\{0\} \cup\left\{\frac{1}{n}: n \in \mathbb{N}\right\}$ be its closure. The following inequalities will be needed later.

Lemma 3.2. (i) Let $\lambda \in \mathbb{C} \backslash \Sigma_{0}$ and set $\alpha:=\operatorname{Re}\left(\frac{1}{\lambda}\right)$. Then there exist constants $d>0$ and $D>0$ (depending on $\alpha$ ) such that

$$
\frac{d}{n^{\alpha}} \leq \prod_{k=1}^{n}\left|1-\frac{1}{k \lambda}\right| \leq \frac{D}{n^{\alpha}}, \quad n \in \mathbb{N}
$$


(ii) For each $m \in \mathbb{N}$ we have that

$$
\frac{(n-1) !}{(n-m) !} \simeq n^{m-1}, \quad \text { for all large } n \in \mathbb{N}
$$

(iii) Let $1<p<\infty$ and $w=(w(n))_{n \in \mathbb{N}}$ be a positive, decreasing sequence. Then

$$
\left(n^{m} w(n)\right)_{n \in \mathbb{N}} \in \ell_{p}, \quad \forall m \in \mathbb{N},
$$

if and only if

$$
\left(n^{m} w(n)^{1 / p}\right)_{n \in \mathbb{N}} \in \ell_{p}, \quad \forall m \in \mathbb{N},
$$

Proof. (i) The inequalities in (8) follow as in the proof of Lemma 7 in [19], where the restriction $\alpha<1$ is assumed. Indeed, with $\frac{1}{\lambda}=\alpha+i \beta$ (for $\alpha, \beta \in \mathbb{R})$ and using $1+x \leq e^{x}$ for $x>0$, we have

$$
\begin{aligned}
& \prod_{k=1}^{n}\left|1-\frac{1}{k \lambda}\right|=\prod_{k=1}^{n}\left(1-\frac{2 \alpha}{k}+\frac{\alpha^{2}+\beta^{2}}{k^{2}}\right)^{1 / 2} \\
& \leq \exp \sum_{k=1}^{n}\left(-\frac{\alpha}{k}+\frac{C}{k^{2}}\right) \leq \exp (-\alpha \log (n)+v) \leq \frac{D}{n^{\alpha}}
\end{aligned}
$$

An application of Taylor's formula to $x \mapsto(1+x)^{-1 / 2}$, for $x>-1$, yields

$$
\begin{aligned}
& \prod_{k=1}^{n}\left|1-\frac{1}{k \lambda}\right|^{-1}=\prod_{k=1}^{n}\left(1-\frac{2 \alpha}{k}+\frac{\alpha^{2}+\beta^{2}}{k^{2}}\right)^{-1 / 2} \leq \prod_{k=1}^{n}\left(1+\frac{\alpha}{k}+\frac{C^{\prime}}{k^{2}}\right) \\
& \leq \exp \sum_{k=1}^{n}\left(\frac{\alpha}{k}+\frac{C^{\prime}}{k^{2}}\right) \leq \exp \left(\alpha \log (n)+v^{\prime}\right)=d^{-1} n^{\alpha} .
\end{aligned}
$$

(ii) Fix $m \in \mathbb{N}$. Then, for all large $n>m$, we have

$\frac{(n-1) !}{(n-m) !}=(n-1) \cdot \ldots \cdot(n-m+1)=n^{m-1} \cdot\left(1-\frac{1}{n}\right) \ldots \cdot\left(1-\frac{m-1}{n}\right) \simeq n^{m-1}$.

(iii) Suppose that $(10)$ holds. Fix $m \in \mathbb{N}$. Let $k \in \mathbb{N}$ satisfy $k \geq(2+m p)$. Since $\left(n^{k} w(n)\right)_{n \in \mathbb{N}} \in \ell_{p}$, there exists $N \in \mathbb{N}$ such that

$$
w(n) \leq \frac{1}{n^{k}} \leq \frac{1}{n^{2+m p}}, \quad n>N .
$$

It follows that

$$
\sum_{n=1}^{\infty} n^{m p} w(n) \leq \sum_{n=1}^{N} n^{m p} w(n)+\sum_{n=N+1}^{\infty} n^{m p}\left(\frac{1}{n^{2+m p}}\right)<\infty,
$$

that is, $\left(n^{m} w(n)^{1 / p}\right)_{n \in \mathbb{N}} \in \ell_{p}$. Accordingly, (11) is satisfied. 
Conversely, suppose that (11) holds. Since $\left(n w(n)^{1 / p}\right)_{n \in \mathbb{N}} \in \ell_{p}$, there exists $K \in \mathbb{N}$ such that $w(n) \leq 1$ for $n \geq K$ and hence, $w(n) \leq w(n)^{1 / p}$ for $n \geq K$. Fix $m \in \mathbb{N}$. Then $n^{m} w(n) \leq n^{m} w(n)^{1 / p}$ for $n \geq K$. Since $\left(n^{m} w(n)^{1 / p}\right)_{n \in \mathbb{N}} \in \ell_{p}$, we can conclude that also $\left(n^{m} w(n)\right)_{n \in \mathbb{N}} \in \ell_{p}$. Hence, (10) is satisfied.

If $S_{w}(p) \neq \emptyset$, then $s_{p} \geq 1$ and so $\frac{p^{\prime}}{2 s_{p}} \leq \frac{p^{\prime}}{2}$, which is relevant for the following results. Also relevant is that $\left\|\mathrm{C}^{(p, w}\right\|<p^{\prime}$ is possible; see Section 2 .

We now come to the main result of this section.

THEOREM 3.3. Let $w=(w(n))_{n \in \mathbb{N}}$ be a positive, decreasing sequence.

(i) Suppose that $S_{w}(p) \neq \emptyset$ for some $1<p<\infty$. Then for the dual operator $\left(\mathrm{C}^{(p, w)}\right)^{\prime} \in \mathcal{L}\left(\left(\ell_{p}(w)\right)^{\prime}\right)$ of $\mathrm{C}^{(p, w)}$ we have

$$
\left\{\lambda \in \mathbb{C}:\left|\lambda-\frac{p^{\prime}}{2 s_{p}}\right|<\frac{p^{\prime}}{2 s_{p}}\right\} \cup \Sigma \subseteq \sigma_{p t}\left(\left(\mathbb{C}^{(p, w)}\right)^{\prime}\right)
$$

and

$$
\sigma_{p t}\left(\left(\mathrm{C}^{(p, w)}\right)^{\prime}\right) \backslash \Sigma \subseteq\left\{\lambda \in \mathbb{C}:\left|\lambda-\frac{p^{\prime}}{2 s_{p}}\right| \leq \frac{p^{\prime}}{2 s_{p}}\right\}
$$

For the Cesàro operator $\mathrm{C}^{(p, w)}$ itself we have

$$
\left\{\lambda \in \mathbb{C}:\left|\lambda-\frac{p^{\prime}}{2 s_{p}}\right| \leq \frac{p^{\prime}}{2 s_{p}}\right\} \cup \Sigma \subseteq \sigma\left(\mathrm{C}^{(p, w)}\right)
$$

and

$$
\sigma\left(\mathrm{C}^{(p, w)}\right) \subseteq\left\{\lambda \in \mathbb{C}:\left|\lambda-\frac{p^{\prime}}{2}\right| \leq \frac{p^{\prime}}{2}\right\} \cap\left\{\lambda \in \mathbb{C}:|\lambda| \leq\left\|\mathbb{C}^{(p, w)}\right\|\right\} .
$$
have

(ii) Suppose that $R_{w} \neq \mathbb{R}$, i.e., $t_{0}<\infty$. Then, for every $1<p<\infty$, we $\left\{\frac{1}{m}: m \in \mathbb{N}, 1 \leq m<\frac{t_{0}}{p}+1\right\} \subseteq \sigma_{p t}\left(C^{(p, w)}\right) \subseteq\left\{\frac{1}{m}: m \in \mathbb{N}, 1 \leq m \leq \frac{t_{0}}{p}+1\right\}$.

If $R_{w}=\mathbb{R}$, then

$$
\sigma_{p t}\left(\mathrm{C}^{(p, w)}\right)=\Sigma, \quad \forall 1<p<\infty .
$$

Proof. The proof is via a series of steps.

(i) By Proposition 2.2 we have $\mathrm{C}^{(p, w)} \in \mathcal{L}\left(\ell_{p}(w)\right)$ with $\left\|\mathrm{C}^{(p, w)}\right\| \leq p^{\prime}$. The dual operator $A:=\left(\mathrm{C}^{p, w}\right)^{\prime} \in \mathcal{L}\left(\ell_{p^{\prime}}\left(w^{-p^{\prime} / p}\right)\right)$ also satisfies $\|A\| \leq p^{\prime}$ and is given by

$$
A y=\left(\sum_{k=n}^{\infty} \frac{y_{k}}{k}\right)_{n \in \mathbb{N}}, \quad y=\left(y_{n}\right)_{n \in \mathbb{N}} \in \ell_{p^{\prime}}\left(w^{-p^{\prime} / p}\right) .
$$


Step 1. $0 \notin \sigma_{p t}(A)$.

Observe that $A y=0$, for some $y \in \ell_{p^{\prime}}\left(w^{-p^{\prime} / p}\right)$, implies that $z_{n}:=$ $\sum_{k=n}^{\infty} \frac{y_{k}}{k}=0$ for all $n \in \mathbb{N}$. Hence, $y_{n}=n\left(z_{n}-z_{n+1}\right)=0$, for $n \in \mathbb{N}$, and so $A$ is injective.

Step 2. $\Sigma \subseteq \sigma_{p t}(A)$.

Let $\lambda \in \Sigma$, i.e., $\lambda=\frac{1}{m}$ for some $m \in \mathbb{N}$. Via (19) below, the non-zero vector $y=\left(y_{n}\right)_{n \in \mathbb{N}}$ defined via $y_{1} \in \mathbb{C} \backslash\{0\}$ arbitrary, $y_{n}:=y_{1} \prod_{k=1}^{n-1}\left(1-\frac{1}{\lambda k}\right)$ for $1<n \leq m$ and $y_{n}:=0$ for $n>m$, which belongs to $\ell_{p^{\prime}}\left(w^{-p^{\prime} / p}\right)$, satisfies $A y=\lambda y$.

Step 3. $\left\{\lambda \in \mathbb{C}:\left|\lambda-\frac{p^{\prime}}{2 s_{p}}\right|<\frac{p^{\prime}}{2 s_{p}}\right\} \subseteq \sigma_{p t}(A)$.

Let $\lambda \in \mathbb{C} \backslash\{0\}$. Then $A y=\lambda y$ for some non-zero $y \in \ell_{p^{\prime}}\left(w^{-p^{\prime} / p}\right)$ if, and only if, $\lambda y_{n}=\sum_{k=n}^{\infty} \frac{y_{k}}{k}$ for all $n \in \mathbb{N}$. This yields, for every $n \in \mathbb{N}$, that $\lambda\left(y_{n}-y_{n+1}\right)=\frac{y_{n}}{n}$ and so $y_{n+1}=\left(1-\frac{1}{\lambda n}\right) y_{n}$. It follows that

$$
y_{n+1}=y_{1} \prod_{k=1}^{n}\left(1-\frac{1}{\lambda k}\right), \quad n \in \mathbb{N}
$$

with $y_{1} \neq 0$. In particular, each eigenvalue of $A$ is simple.

Let now $\lambda \in \mathbb{C} \backslash \sum$ satisfy $\left|\lambda-\frac{p^{\prime}}{2 s_{p}}\right|<\frac{p^{\prime}}{2 s_{p}}$ (equivalently, $\alpha:=\operatorname{Re}\left(\frac{1}{\lambda}\right)>\frac{s_{p}}{p^{\prime}}$, i.e., $\left.\alpha p^{\prime}=\operatorname{Re}\left(\frac{p^{\prime}}{\lambda}\right)>s_{p}\right)$. For such a $\lambda$ the vector $y=\left(y_{n}\right)_{n \in \mathbb{N}} \in \mathbb{C}^{\mathbb{N}}$ defined by (19) actually belongs to $\ell_{p^{\prime}}\left(w^{-p^{\prime} / p}\right)$. Indeed, via Lemma 3.2(i) there exists $c=c(\lambda)>0$ such that

$$
\prod_{k=1}^{n}\left|1-\frac{1}{\lambda k}\right|^{p^{\prime}} \leq c n^{-\operatorname{Re}\left(p^{\prime} / \lambda\right)}, \quad n \in \mathbb{N} .
$$

It then follows from (19) that

$$
\begin{aligned}
\sum_{n=1}^{\infty}\left|y_{n}\right|^{p^{\prime}} w(n)^{-p^{\prime} / p} & =\left|y_{1}\right|^{p^{\prime}} w(1)^{-p^{\prime} / p}+\left|y_{1}\right|^{p^{\prime}} \sum_{n=2}^{\infty} \prod_{k=1}^{n}\left|1-\frac{1}{\lambda k}\right|^{p^{\prime}} w(n)^{-p^{\prime} / p} \\
& \leq\left|y_{1}\right|^{p^{\prime}} w(1)^{-p^{\prime} / p}+c\left|y_{1}\right|^{p^{\prime}} \sum_{n=2}^{\infty} n^{-\operatorname{Re}\left(p^{\prime} / \lambda\right)} w(n)^{-p^{\prime} / p},
\end{aligned}
$$

where the series $\sum_{n=2}^{\infty} n^{-\operatorname{Re}\left(p^{\prime} / \lambda\right)} w(n)^{-p^{\prime} / p}$ converges because $\operatorname{Re}\left(p^{\prime} / \lambda\right) \in$ $S_{w}(p)$, that is, $y \in \ell_{p^{\prime}}\left(w^{-p^{\prime} / p}\right)$. Hence, $\lambda \in \sigma_{p t}(A)$.

Step 4. $\sigma_{p t}(A) \backslash \Sigma_{0} \subseteq\left\{\lambda \in \mathbb{C}:\left|\lambda-\frac{p^{\prime}}{2 s_{p}}\right| \leq \frac{p^{\prime}}{2 s_{p}}\right\}$.

Fix $\lambda \in \sigma_{p t}(A) \backslash \Sigma_{0}$. According to (8) there exists $\beta=\beta(\lambda)>0$ such that

$$
\prod_{k=1}^{n}\left|1-\frac{1}{\lambda k}\right|^{p^{\prime}} \geq \beta \cdot n^{-\operatorname{Re}\left(p^{\prime} / \lambda\right)}, \quad n \in \mathbb{N} .
$$


But, as argued in Step 2 (for any $y_{1} \in \mathbb{C} \backslash\{0\}$ ) the eigenvector $y=$ $\left(y_{n}\right)_{n \in \mathbb{N}}$ corresponding to the eigenvalue $\lambda$ of $A$, which necessarily belongs to $\ell_{p^{\prime}}\left(w^{-p^{\prime} / p}\right)$, i.e., $\sum_{n=1}^{\infty}\left|y_{n}\right|^{p^{\prime}} w(n)^{-p^{\prime} / p}<\infty$, is given by (19). Then (20) implies that also $\sum_{n=1}^{\infty} \frac{1}{n^{\operatorname{Re}\left(p^{\prime} / \lambda\right)} w(n)^{p^{\prime} / p}}<\infty$, i.e., $\operatorname{Re}\left(\frac{p^{\prime}}{\lambda}\right) \in S_{w}(p)$ and so $\operatorname{Re}\left(\frac{p^{\prime}}{\lambda}\right) \geq s_{p}$. Equivalently, $\operatorname{Re}\left(\frac{1}{\lambda}\right) \geq \frac{s_{p}}{p^{\prime}}$, i.e., $\lambda \in\left\{\mu \in \mathbb{C}:\left|\mu-\frac{p^{\prime}}{2 s_{p}}\right| \leq \frac{p^{\prime}}{2 s_{p}}\right\}$.

It is clear that Steps 1-4 establish the two containments in (12) and (13).

For every $T \in \mathcal{L}(X)$ with $X$ a Banach space, it is known that $\sigma_{p t}\left(T^{\prime}\right) \subseteq$ $\sigma(T),[10$, p.581], with $\sigma(T)$ a closed subset of $\mathbb{C}$. Accordingly, (14) follows from $(12)$.

Step 5. $\sigma\left(C^{(p, w)}\right) \subseteq\left\{\lambda \in \mathbb{C}:\left|\lambda-\frac{p^{\prime}}{2}\right| \leq \frac{p^{\prime}}{2}\right\}$.

It suffices to show that every $\lambda \in \mathbb{C}$ with $\left|\lambda-\frac{p^{\prime}}{2}\right|>\frac{p^{\prime}}{2}$ belongs to $\rho\left(\mathbf{C}^{(p, w)}\right)$. To do this we argue as in [7]. We recall the formula for (C $\lambda I)^{-1}: \mathbb{C}^{\mathbb{N}} \rightarrow \mathbb{C}^{\mathbb{N}}$ whenever $\lambda \notin \Sigma_{0},[19$, p.266]. For $n \in \mathbb{N}$ the $n$-th row of the matrix for $(\mathrm{C}-\lambda I)^{-1}$ has the entries

$$
\begin{gathered}
\frac{-1}{n \lambda^{2} \prod_{k=m}^{n}\left(1-\frac{1}{\lambda k}\right)}, \quad 1 \leq m<n, \\
\frac{n}{1-n \lambda}=\frac{1}{\frac{1}{n}-\lambda}, \quad m=n,
\end{gathered}
$$

and all the other entries in row $n$ are equal to 0 . So, we can write

$$
(\mathrm{C}-\lambda I)^{-1}=D_{\lambda}-\frac{1}{\lambda^{2}} E_{\lambda}
$$

where the diagonal operator $D_{\lambda}=\left(d_{n m}\right)_{n, m \in \mathbb{N}}$ is given by $d_{n n}:=\frac{1}{\frac{1}{n}-\lambda}$ and $d_{n m}:=0$ if $n \neq m$. The operator $E_{\lambda}=\left(e_{n m}\right)_{n, m \in \mathbb{N}}$ is then the lower triangular matrix with $e_{1 m}=0$ for all $m \in \mathbb{N}$, and for every $n \geq 2$ with $e_{n m}:=\frac{1}{n \prod_{k=m}^{n}\left(1-\frac{1}{\lambda k}\right)}$ if $1 \leq m<n$ and $e_{n m}:=0$ if $m \geq n$.

If $\lambda \notin \Sigma_{0}$, then $d(\lambda):=\operatorname{dist}\left(\lambda, \Sigma_{0}\right)>0$ and $\left|d_{n n}\right| \leq \frac{1}{d(\lambda)}$ for $n \in \mathbb{N}$. Hence, for every $x \in \ell_{p}(w)$, we have

$$
\begin{aligned}
\left\|D_{\lambda}(x)\right\|_{p, w} & =\left(\sum_{n=1}^{\infty}\left|d_{n n} x_{n}\right|^{p} w(n)\right)^{1 / p} \\
& \leq \frac{1}{d(\lambda)}\left(\sum_{n=1}^{\infty}\left|x_{n}\right|^{p} w(n)\right)^{1 / p}=\frac{1}{d(\lambda)}\|x\|_{p, w} .
\end{aligned}
$$

This means that $D_{\lambda} \in \mathcal{L}\left(\ell_{p}(w)\right)$. So, by (21) it remains to show that $E_{\lambda} \in$ $\mathcal{L}\left(\ell_{p}(w)\right)$ whenever $\lambda \in \mathbb{C}$ satisfies $\left|\lambda-\frac{p^{\prime}}{2}\right|>\frac{p^{\prime}}{2}$. To this end, we note that 
if $\lambda \in \mathbb{C} \backslash \Sigma_{0}$ then, with $\alpha:=\operatorname{Re}\left(\frac{1}{\lambda}\right)$, it follows from (8) that

$$
\begin{aligned}
& \left|e_{n 1}\right| \leq \frac{d^{-1}}{n^{1-\alpha}}, \quad n \geq 2, \\
& \left|e_{n m}\right| \leq \frac{d^{-1} D^{\prime}}{n^{1-\alpha} m^{\alpha}}, \quad 2 \leq m<n,
\end{aligned}
$$

for some constants $d>0$ and $D^{\prime}>0$ depending on $\lambda$. So, for every $\lambda \in \mathbb{C} \backslash \Sigma_{0}$ there exists $c=c(\lambda)>0$ such that

$$
\left|\left(E_{\lambda}(x)\right)_{n}\right| \leq c\left(G_{\lambda}(|x|)\right)_{n}, \quad x \in \mathbb{C}^{\mathbb{N}}, n \in \mathbb{N},
$$

where $\left(G_{\lambda}(x)\right)_{n}:=\sum_{k=1}^{n} \frac{x_{k}}{n^{1-\alpha} k^{\alpha}}$ with $\alpha:=\operatorname{Re}\left(\frac{1}{\lambda}\right)$ and for all $x \in \mathbb{C}^{\mathbb{N}}$ and $n \in \mathbb{N}$. Clearly (22) implies that $E_{\lambda} \in \mathcal{L}\left(\ell_{p}(w)\right)$ whenever $G_{\lambda} \in \mathcal{L}\left(\ell_{p}(w)\right)$.

Claim: $G_{\lambda} \in \mathcal{L}\left(\ell_{p}(w)\right)$ whenever $\lambda \in \mathbb{C}$ satisfies $\left|\lambda-\frac{p^{\prime}}{2}\right|>\frac{p^{\prime}}{2}$.

To establish this claim fix $\lambda \in \mathbb{C}$ with $\left|\lambda-\frac{p^{\prime}}{2}\right|>\frac{p^{\prime}}{2}$. Then necessarily $\lambda \notin \Sigma_{0}$ with $\alpha:=\operatorname{Re}\left(\frac{1}{\lambda}\right)<\frac{1}{p^{\prime}}$ and so $(1-\alpha) p>1$. This implies that $\alpha<1$. Observe that $G_{\lambda} \in \mathcal{L}\left(\ell_{p}(w)\right)$ if, and only if, the operator $\tilde{G}_{\lambda}: \mathbb{C}^{\mathbb{N}} \rightarrow \mathbb{C}^{\mathbb{N}}$ given by

$$
\left(\tilde{G}_{\lambda}(x)\right)_{n}=w(n)^{1 / p} \sum_{k=1}^{n} \frac{w(k)^{-1 / p}}{n^{1-\alpha} k^{\alpha}} x_{k}, \quad x \in \mathbb{C}^{\mathbb{N}}, n \in \mathbb{N},
$$

defines a continuous linear operator on $\ell_{p}$ (the proof of this is along the lines of that of Lemma 2.1). To prove that indeed $\tilde{G}_{\lambda} \in \mathcal{L}\left(\ell_{p}\right)$ we need to distinguish the three cases; a) $\alpha=0$; b) $\alpha<0$ and c) $0<\alpha<1$ and establish relevant inequalities in each case.

Case a). Since $w$ is decreasing, we have, for every $n \in \mathbb{N}$, that

$$
\sum_{k=1}^{n} \frac{1}{w(k)^{1 /(p-1)} k^{\alpha p /(p-1)}}=\sum_{k=1}^{n} \frac{1}{w(k)^{1 /(p-1)}} \leq \frac{n}{w(n)^{1 /(p-1)}}
$$

and hence, for every $m \in \mathbb{N}$, that

$$
\sum_{n=1}^{m}\left(\frac{w(n)^{1 / p}}{n} \sum_{k=1}^{n} \frac{1}{w(k)^{1 /(p-1)}}\right)^{p} \leq \sum_{n=1}^{m} \frac{1}{w(n)^{1 /(p-1)}} .
$$

Case b). Observe, for every $n \in \mathbb{N}$, that

$$
\begin{aligned}
& \sum_{k=1}^{n} \frac{1}{w(k)^{1 /(p-1)} k^{\alpha p /(p-1)}} \leq \frac{1}{w(n)^{1 /(p-1)}} \int_{1}^{n+1} x^{-\alpha p /(p-1)} d x \\
& =\frac{1}{w(n)^{1 /(p-1)}} \frac{\left((n+1)^{-\frac{\alpha p}{p-1}+1}-1\right)}{-\frac{\alpha p}{p-1}+1} \leq \frac{(p-1)}{(p(1-\alpha)-1)} \frac{(n+1)^{\frac{p(1-\alpha)-1}{p-1}}}{w(n)^{1 /(p-1)}} .
\end{aligned}
$$


Setting $c:=\frac{p-1}{p(1-\alpha)-1}>0$ it follows, for every $m \in \mathbb{N}$, that

$$
\begin{aligned}
& \sum_{n=1}^{m}\left(\frac{w(n)^{1 / p}}{n^{1-\alpha}} \sum_{k=1}^{n} \frac{1}{w(k)^{1 /(p-1)} k^{\alpha p /(p-1)}}\right)^{p} \leq c^{p} \sum_{n=1}^{m} \frac{(n+1)^{\frac{p[p(1-\alpha)-1]}{p-1}}}{w(n)^{1 /(p-1)} n^{(1-\alpha) p}} \\
& \quad \leq 2^{\frac{p(p(1-\alpha)-1)}{p-1}} c^{p} \sum_{n=1}^{m} \frac{1}{w(n)^{1 /(p-1)} n^{\alpha p /(p-1)}} .
\end{aligned}
$$

Case c). We have, for every $n \in \mathbb{N}$, still with $c=\frac{p-1}{p(1-\alpha)-1}$, that

$$
\begin{aligned}
& \sum_{k=2}^{n} \frac{1}{w(k)^{1 /(p-1)} k^{\alpha p /(p-1)}} \leq \frac{1}{w(n)^{1 /(p-1)}} \int_{1}^{n} \frac{1}{x^{\alpha p /(p-1)}} d x \\
& \quad=\frac{c}{w(n)^{1 /(p-1)}}\left(n^{\frac{p(1-\alpha)-1}{p-1}}-1\right) .
\end{aligned}
$$

Since $(1-\alpha) p>1$ (i.e., $(1-\alpha) p-1>0)$ and $\alpha p>0$ with $\frac{1}{w(1)} \leq \frac{1}{w(n)}$, this implies, for every $n \in \mathbb{N}$, that

$$
\begin{aligned}
& \left(\frac{w(n)^{1 / p}}{n^{1-\alpha}} \sum_{k=1}^{n} \frac{1}{w(k)^{1 /(p-1)} k^{\alpha p /(p-1)}}\right)^{p} \\
& \leq\left[\frac{w(n)^{1 / p}}{n^{1-\alpha} w(1)^{1 /(p-1)}}+\frac{w(n)^{1 / p} c}{n^{1-\alpha} w(n)^{1 /(p-1)}}\left(n^{\frac{p(1-\alpha)-1}{p-1}}-1\right)\right]^{p} \\
& \leq\left[\frac{w(n)^{1 / p}}{n^{1-\alpha} w(n)^{1 /(p-1)}}+\frac{w(n)^{1 / p} c}{n^{1-\alpha} w(n)^{1 /(p-1)}}\left(n^{\frac{p(1-\alpha)-1}{p-1}}-1\right)\right]^{p} \\
& \quad=\left[(1-c) \frac{w(n)^{1 / p}}{n^{1-\alpha} w(n)^{1 /(p-1)}}+\frac{w(n)^{1 / p} c}{n^{1-\alpha} w(n)^{1 /(p-1)}} n^{\frac{p(1-\alpha)-1}{p-1}}\right]^{p} \\
& \quad=\left(\frac{-\alpha p}{p(1-\alpha)-1} \frac{w(n)^{1 / p}}{n^{1-\alpha} w(n)^{1 /(p-1)}}+\frac{w(n)^{-1 / p(p-1)} c}{n^{1-\alpha}} n^{\frac{p(1-\alpha)-1}{p-1}}\right)^{p} \\
& \leq\left(\frac{w(n)^{-1 / p(p-1)} c}{n^{1-\alpha}} n^{\frac{p(1-\alpha)-1}{p-1}}\right)^{p} \\
& =c^{p} w(n)^{-1 /(p-1)} n^{-\alpha p /(p-1)} .
\end{aligned}
$$

Hence, for every $m \in \mathbb{N}$, we have that

$$
\sum_{n=1}^{m}\left(\frac{w(n)^{1 / p}}{n^{1-\alpha}} \sum_{k=1}^{n} \frac{1}{w(k)^{1 /(p-1)} k^{\alpha p /(p-1)}}\right)^{p} \leq c^{p} \sum_{n=1}^{m} \frac{1}{w(n)^{1 /(p-1)} n^{\alpha p /(p-1)}} .
$$

The inequalities (23), (24) and (25) imply that $\tilde{G}_{\lambda} \in \mathcal{L}\left(\ell_{p}\right)$; indeed, in each case, suitable choices of $a_{n}$ and $b_{k}$ (with $p=q$ ) allow us to apply Theorem 2(ii) of [5]. This establishes the claim and hence, also Step 5. 
Step 6. $\sigma\left(\mathrm{C}^{(p, w)}\right) \subseteq\left\{\lambda \in \mathbb{C}:|\lambda| \leq\left\|\mathrm{C}^{(p, w)}\right\|\right\}$.

This is well known, [10, Ch.VII Lemma 3.4].

Steps 5 and 6 clearly yield (15). The proof of part (i) is thereby complete.

(ii) Suppose first that $R_{w} \neq \mathbb{R}$. Fix any $1<p<\infty$.

Step 7. Both of the inclusions in (16) are valid.

The Cesàro operator $\mathrm{C}^{(p, w)}$ is clearly injective. So, $0 \notin \sigma_{p t}\left(\mathrm{C}^{(p, w)}\right)$. Let $\lambda \in \mathbb{C} \backslash\{0\}$. Consider the equation $(\lambda I-\mathrm{C}) x=0$ with $x=\left(x_{n}\right)_{n \in \mathbb{N}} \in$ $\mathbb{C}^{\mathbb{N}} \backslash\{0\}$. Then $x_{1}=\lambda x_{1}$ and $(2 \lambda-1) x_{2}=x_{1}$ and $(n \lambda-1) x_{n}=\lambda(n-1) x_{n-1}$ for all $n \geq 3$. If $m \in \mathbb{N}$ denotes the smallest positive integer such that $x_{m} \neq 0$, then it follows that $\lambda=\frac{1}{m}$ and so $x_{n}=\frac{n-1}{n-m} x_{n-1}$ for all $n>m$. Thus, we deduce that

$$
x_{n}=x_{m+(n-m)}=\frac{(n-1) !}{(m-1) !(n-m) !} x_{m}, \quad n \geq m .
$$

According to $(9)$ we have $\frac{(n-1) !}{(m-1) !(n-m) !} \simeq \frac{1}{(m-1) !} \cdot n^{m-1}$, for each $m \in \mathbb{N}$. So, $x \in \ell_{p}(w)$ if, and only if, the series $\sum_{n=m+1}^{\infty} n^{(m-1) p} w(n)$ converges. But, the series $\sum_{n=m+1}^{\infty} n^{(m-1) p} w(n)$ converges precisely when $(m-1) p \in R_{w}$. In this case, $(m-1) p \leq t_{0}$, i.e., $m \leq \frac{t_{0}}{p}+1$. So, $\sigma_{p t}\left(\mathrm{C}^{(p, w)}\right) \subseteq\left\{\frac{1}{m}: m \in \mathbb{N}, 1 \leq\right.$ $\left.m \leq \frac{t_{0}}{p}+1\right\}$.

Conversely, if $m<\frac{t_{0}}{p}+1$ for some $m \in \mathbb{N}$, i.e., $(m-1) p<t_{0}$, then $(m-1) p \in R_{w}$ as $t_{0}=\sup R_{w}$. Then the vector $x \in \mathbb{C}^{\mathbb{N}}$ defined according to (26), with $x_{1}=\ldots=x_{m-1}=0$ and for any arbitrary $x_{m} \neq 0$, belongs to $\ell_{p}(w)$. Therefore, $\frac{1}{m} \in \sigma_{p t}\left(\mathrm{C}^{(p, w)}\right)$.

Step 8. Assume now that $R_{w}=\mathbb{R}$. Then (17) is valid.

Fix $1<p<\infty$. As argued in Step 7, the point $\frac{1}{m} \in \sigma_{p t}\left(\mathrm{C}^{(p, w)}\right)$ if and only if $(m-1) p \in R_{w}$. But, for $R_{w}=\mathbb{R}$, this is satisfied for every $m \in \mathbb{N}$ and so $\Sigma \subseteq \sigma_{p t}\left(\mathrm{C}^{(p, w)}\right)$. On the other hand, it is also shown in the proof of Step 7 that every eigenvalue $\lambda$ of $C: \mathbb{C}^{\mathbb{N}} \rightarrow \mathbb{C}^{\mathbb{N}}$ must have the form $\lambda=\frac{1}{m}$ for some $m \in \mathbb{N}$. Since every eigenvalue of $C^{(p, w)}$ is also an eigenvalue of $C$ $\left(\right.$ as $\left.\ell_{p}(w) \subseteq \mathbb{C}^{\mathbb{N}}\right)$, it follows that $\sigma_{p t}\left(\mathrm{C}^{(p, w)}\right) \subseteq \Sigma$.

REMARK 1. (i) If $s_{p} \notin S_{w}(p)$, for some $1<p<\infty$, then the argument of Step 4 in the proof of Theorem 3.3 implies that (12) reduces to the equality

$$
\sigma_{p t}\left(\left(\mathrm{C}^{(p, w)}\right)^{\prime}\right)=\left\{\lambda \in \mathbb{C}:\left|\lambda-\frac{p^{\prime}}{2 s_{p}}\right|<\frac{p^{\prime}}{2 s_{p}}\right\} \cup \Sigma .
$$

Also, if $t_{0} \notin R_{w}$, then (16) reduces to the equality

$$
\sigma_{p t}\left(\mathrm{C}^{(p, w)}\right)=\left\{\frac{1}{m}: m \in \mathbb{N}, 1 \leq m<\frac{t_{0}}{p}+1\right\}, 1<p<\infty .
$$

(ii) For $w(n)=1$ for all $n \in \mathbb{N}$, in which case $\ell_{p}(w)=\ell_{p}$ and $s_{p}=1$, we have that $\mathrm{C}^{(p, w)}=\mathrm{C}^{(p)}$ for all $1<p<\infty$ with $\left\|\mathrm{C}^{(p, w)}\right\|=\left\|\mathrm{C}^{(p)}\right\|=p^{\prime}$. Then 
(14) and (15) imply the known fact that

$$
\sigma\left(\mathrm{C}^{(p)}\right)=\left\{\lambda \in \mathbb{C}:\left|\lambda-\frac{p^{\prime}}{2}\right| \leq \frac{p^{\prime}}{2}\right\}
$$

Since $t_{0}=-1$, we also recover from (16) the known fact that $\sigma_{p t}\left(\mathrm{C}^{(p)}\right)=\emptyset$.

(iii) According to (14), for $w$ positive, decreasing and with $S_{w}(p) \neq \emptyset$ we have

$$
\frac{p^{\prime}}{s_{p}} \leq \max \left\{1, \frac{p^{\prime}}{s_{p}}\right\} \leq\left\|\mathrm{C}^{(p, w)}\right\| \leq p^{\prime} .
$$

In particular, whenever $s_{p}=1$ (see e.g., Example 3(i) below), the inequalities in (28) imply that necessarily $\left\|C^{(p, w)}\right\|=p^{\prime}$ is as large as possible.

For the special case when $w(n)=\frac{1}{n^{\alpha}}, n \in \mathbb{N}$, for some $\alpha>0$, direct calculation yields that $s_{p}=1+\frac{\alpha p^{\prime}}{p}$ and so $S_{w}(p) \neq \emptyset$ for all $1<p<\infty$. It follows that

$$
\frac{p^{\prime}}{s_{p}}=\frac{p}{\alpha+p-1}=m_{1},
$$

where $m_{1}$ occurs in the lower bound for $\left\|\mathrm{C}^{(p, w)}\right\|$ as given in (5); see Proposition 2.3. Hence, $(28)$ yields that $m_{1} \leq\left\|\mathrm{C}^{(p, w)}\right\|$. Combined with Example 1 (iii) we can conclude that

$$
\max \left\{m_{1}, m_{2}\right\} \leq\left\|\mathrm{C}^{(p, w)}\right\| .
$$

This provides an alternate proof, to that in [12], of the same estimate in (5).

(iv) An examination of the argument for Step 2 in the proof of Theorem 3.3 (i) shows that the assumption $S_{w}(p) \neq \emptyset$ is not used there, i.e., we always have

$$
\Sigma \subseteq \sigma_{p t}\left(\left(\mathrm{C}^{(p, w)}\right)^{\prime}\right)
$$

for every $1<p<\infty$ and every positive, decreasing weight $w$.

We now present some relevant examples.

Example 3. (i) Suppose that $w(n)=\frac{1}{(\log (n+1))^{\gamma}}$ for $n \in \mathbb{N}$ with $\gamma \geq 0$. Then $\sum_{n=1}^{\infty} \frac{1}{n^{s} w(n)^{p^{\prime} / p}}<\infty$ if and only if $s>1$ and hence, $s_{p}=1$ for every $1<p<\infty$. In view of Remark 1 (iii) we have that $\left\|C^{(p, w)}\right\|=p^{\prime}$. Moreover, $\sum_{n=1}^{\infty} n^{t} w(n)<\infty$ if and only if $t<-1$ or $t \leq-1$ in case $\gamma>1$. Hence, $t_{0}=-1$. According to Theorem 3.3 we have, for each $1<p<\infty$, that

$$
\sigma\left(\mathrm{C}^{(p, w)}\right)=\left\{\lambda \in \mathbb{C}:\left|\lambda-\frac{p^{\prime}}{2}\right| \leq \frac{p^{\prime}}{2}\right\}, \quad \sigma_{p t}\left(\mathrm{C}^{(p, w)}\right)=\emptyset .
$$

In particular, equality may occur in (15). For the case when $\gamma=0$ (so that $w(n)=1$ for $n \in \mathbb{N}$ ), we recover the known result about the spectrum of $\mathrm{C}^{(p)} \in \mathcal{L}\left(\ell_{p}\right)$, for $1<p<\infty,[6],[15]$. 
(ii) More generally, suppose that $w(n)=\frac{1}{n^{\beta}(\log (n+1))^{\gamma}}$ for $n \in \mathbb{N}$ with $\beta \geq 0$ and $\gamma \geq 0$. Then $\sum_{n=1}^{\infty} \frac{1}{n^{s} w(n)^{p^{\prime} / p}}<\infty$ if and only if $s>\beta \frac{p^{\prime}}{p}+1$ and so $s_{p}=\beta \frac{p^{\prime}}{p}+1$ for every $1<p<\infty$. Moreover, $\sum_{n=1}^{\infty} n^{t} w(n)<\infty$ if and only if $t<(\beta-1)$ or $t \leq(\beta-1)$ in case $\gamma>1$. Hence, $t_{0}=\beta-1$. According to Theorem 3.3 we have, for each $1<p<\infty$, that

$$
\left\{\lambda \in \mathbb{C}:\left|\lambda-\frac{p^{\prime}}{2\left(\left(\beta p^{\prime} / p\right)+1\right)}\right| \leq \frac{p^{\prime}}{2\left(\left(\beta p^{\prime} / p\right)+1\right)}\right\} \cup \Sigma \subseteq \sigma\left(\mathrm{C}^{(p, w)}\right)
$$

and

$$
\sigma_{p t}\left(\mathbf{C}^{(p, w)}\right)=\left\{\frac{1}{m}: m \in \mathbb{N}, 1 \leq m<\frac{\beta-1}{p}+1\right\} .
$$

In particular, $\sigma_{p t}\left(\mathrm{C}^{(p, w)}\right)=\emptyset$ whenever $\beta \in[0,1]$. We claim that actually

$$
\left\{\lambda \in \mathbb{C}:\left|\lambda-\frac{p^{\prime}}{2\left(\left(\beta p^{\prime} / p\right)+1\right)}\right| \leq \frac{p^{\prime}}{2\left(\left(\beta p^{\prime} / p\right)+1\right)}\right\} \cup \Sigma=\sigma\left(\mathrm{C}^{(p, w)}\right),
$$

which shows that equality may occur in (14).

Keeping in mind the argument for Step 5 in the proof of Theorem 3.3, to verify (29) it suffices to prove that every $\lambda \in \mathbb{C} \backslash\{0\}$ satisfying $\left|\lambda-\frac{p^{\prime}}{2\left(\left(\beta p^{\prime} / p\right)+1\right)}\right|>$ $\frac{p^{\prime}}{2\left(\left(\beta p^{\prime} / p\right)+1\right)}$ belongs to $\rho\left(\mathrm{C}^{(p, w)}\right)$, i.e., that the operator $\tilde{G}_{\lambda} \in \mathcal{L}\left(\ell_{p}\right)$. So, fix such a $\lambda$ and note that $\alpha:=\operatorname{Re}\left(\frac{1}{\lambda}\right)<\left(\beta \frac{p^{\prime}}{p}+1\right) / p^{\prime}=\frac{\beta}{p}+\frac{1}{p^{\prime}}$. We also observe, for our particular $w$, that the operator $\tilde{G}_{\lambda}$ is given by

$$
\left(\tilde{G}_{\lambda}(x)\right)_{n}=\frac{1}{n^{1-\alpha+(\beta / p)} \log ^{\gamma / p}(n+1)} \sum_{k=1}^{n} \frac{x_{k}}{k^{\alpha-(\beta / p)} \log ^{-\gamma / p}(k+1)},
$$

for $x=\left(x_{n}\right)_{n \in \mathbb{N}} \in \mathbb{C}^{\mathbb{N}}$. So, $\tilde{G}_{\lambda}$ is given by the factorable matrix with $a_{n}:=n^{-(1-\alpha+(\beta / p))} \log ^{-\gamma / p}(n+1)$ and $b_{k}:=k^{-(\alpha-(\beta / p))} \log ^{\gamma / p}(k+1)$, where $\alpha<\frac{\beta}{p}+\frac{1}{p^{\prime}}=\frac{\beta}{p}+1-\frac{1}{p}$ implies that $1-\alpha+\frac{\beta}{p}>\frac{1}{p}$ and we have that $\left(1-\alpha+\frac{\beta}{p}\right)+\left(\alpha-\frac{\beta}{p}\right)=1=\frac{1}{p}+\frac{1}{p^{\prime}}$ and also that $\left(\frac{\gamma}{p}\right)+\left(-\frac{\gamma}{p}\right)=0$. According to Corollary 9 (ii) of [5] it follows that $\tilde{G}_{\lambda} \in \mathcal{L}\left(\ell_{p}\right)$ and the claim is proved.

Finally, since $s_{p}=\frac{\beta+p-1}{p-1}$, it follows from (28) that

$$
p^{\prime} \cdot \frac{p-1}{\beta+p-1} \leq\left\|\mathrm{C}^{(p, w)}\right\| \leq p^{\prime}, \quad 1<p<\infty,
$$

with $\frac{p-1}{\beta+p-1} \uparrow 1$ for $\beta \downarrow 0$. This example also shows that the inequality $t_{0} \leq \frac{s_{p} p^{\prime}}{p}$ (cf. Proposition 3.1(i)) can be strict. For $\beta \downarrow 0$ it follows from (14) and (15) that

$$
\sigma\left(\mathrm{C}^{(p, w)}\right) \uparrow\left\{\lambda \in \mathbb{C}:\left|\lambda-\frac{p^{\prime}}{2}\right|<\frac{p^{\prime}}{2}\right\},
$$

whose closure equals $\sigma\left(\mathrm{C}^{(p)}\right)=\sigma\left(\mathrm{C}^{(p, w)}\right)$ for $w$ as in (i). 
It is clear from (16) that $C^{(p, w)}$ has at most finitely many eigenvalues whenever $t_{0} \in \mathbb{R}$. The following result characterizes when $\sigma_{p t}\left(C^{(p, w)}\right)$ is an infinite set; see also Remark 2(i) below. Recall that a sequence $u=$ $\left(u_{n}\right)_{n \in \mathbb{N}} \in \mathbb{C}^{\mathbb{N}}$ is rapidly decreasing if $\left(n^{m} u_{n}\right)_{n \in \mathbb{N}} \in \ell_{1}$ for every $m \in \mathbb{N}$. The space of all rapidly decreasing, $\mathbb{C}$-valued sequences is usually denoted by $s$.

Proposition 3.4. Let $w=(w(n))_{n \in \mathbb{N}}$ be a positive, decreasing sequence.

(i) The following assertions are equivalent.

(1) $R_{w}=\mathbb{R}$

(2) $\left(n^{m} w(n)\right)_{n \in \mathbb{N}} \in \ell_{1}$ for all $m \in \mathbb{N}$.

(3) $\left(n^{m} w(n)\right)_{n \in \mathbb{N}} \in c_{0}$ for all $m \in \mathbb{N}$.

(4) $w \in s$.

(ii) For each $1<p<\infty$, the following assertions are equivalent.

(5) $\Sigma \subseteq \sigma_{p t}\left(\mathrm{C}^{(p, w)}\right)$.

(6) $\left(n^{m} w(n)\right)_{n \in \mathbb{N}} \in \ell_{p}$ for all $m \in \mathbb{N}$.

(iii) Any one of the equivalent assertions (1)-(4) implies that both (5) and (6) are valid, for every $1<p<\infty$.

(iv) If (6) holds for some $1<p<\infty$, then each assertion (1)-(4) is satisfied.

Proof. (i) (1) $\Leftrightarrow(2)$ follows from the definition of $R_{w}$.

$(2) \Leftrightarrow(3)$. That $(2) \Rightarrow(3)$ is immediate from $\ell_{1} \subseteq c_{0}$.

Assume (3). Fix $t \in \mathbb{N}$ and set $m=t+2$. Then $\left(n^{m} w(n)\right)_{n \in \mathbb{N}} \in c_{0}$ implies that $\sup _{n \in \mathbb{N}} n^{m} w(n)<\infty$. Accordingly,

$$
\sum_{n=1}^{\infty} n^{t} w(n)=\sum_{n=1}^{\infty} \frac{1}{n^{2}} n^{m} w(n) \leq \frac{\pi^{2}}{6} \sup _{n \in \mathbb{N}} n^{m} w(n)<\infty .
$$

Since $t$ is arbitrary, we can conclude that (2) holds.

$(2) \Leftrightarrow(4)$. Clear from the definition of the space $s$.

(ii) Since $C^{(p, w)}$ is injective, $0 \notin \sigma_{p t}\left(C^{(p, w)}\right)$. By $(9)$ and $(26), \lambda \in \mathbb{C} \backslash$ $\{0\}$ is an eigenvalue of $C^{(p, w)}$ if and only if $\lambda=\frac{1}{m}$ for some $m \in \mathbb{N}$ with the corresponding 1-dimensional eigenspace generated by a vector $x^{[m]}=$ $\left(x_{n}^{[m]}\right)_{n \in \mathbb{N}} \in \mathbb{C}^{\mathbb{N}}$ satisfying $x_{n}^{[m]} \simeq n^{m-1}$. So, $\Sigma \subseteq \sigma_{p t}\left(\mathrm{C}^{(p, w)}\right)$ if and only if $\left(n^{m-1}\right)_{n \in \mathbb{N}} \in \ell_{p}(w)$ for all $m \in \mathbb{N}$, that is, if and only if $\left(n^{m} w(n)^{1 / p}\right)_{n \in \mathbb{N}} \in \ell_{p}$ for all $m \in \mathbb{N}$, which is equivalent to (6) via Lemma 3.2(iii).

(iii) Follows immediately from parts (i) and (ii) and the fact that $(2) \Rightarrow(6)$ since $\ell_{1} \subseteq \ell_{p}$ for every $1<p<\infty$.

(iv) Immediate from $\ell_{p} \subseteq c_{0}$ for every $1<p<\infty$. 
Given a decreasing sequence $w=(w(n))_{n \in \mathbb{N}}$ of positive real numbers, set $\alpha_{n}:=-\log w(n)$, for $n \in \mathbb{N}$. Then $w(n)=e^{-\alpha_{n}}$, for $n \in \mathbb{N}$. Moreover, $\alpha_{n} \rightarrow \infty$ for $n \rightarrow \infty$ if and only if $w(n) \rightarrow 0$ for $n \rightarrow \infty$.

Corollary 3.5. Let $w=(w(n))_{n \in \mathbb{N}}$ be a decreasing, positive sequence.

(i) If $w \in s$, then $\lim _{n \rightarrow \infty} \frac{\log n}{\alpha_{n}}=0$.

(ii) If $\lim _{n \rightarrow \infty} \frac{\log n}{\alpha_{n}}=0$ and $w(N)<1$ for some $N$, then $w \in s$.

Proof. (i) Since $w \in s$, condition (3) in Proposition 3.4 implies that

$$
\forall m \in \mathbb{N} \exists n_{m} \in \mathbb{N} \forall n \geq n_{m}: n^{m} w(n)=\frac{n^{m}}{e^{\alpha_{n}}}<1,
$$

i.e., that

$$
\forall m \in \mathbb{N} \exists n_{m} \in \mathbb{N} \forall n \geq n_{m}: n^{m}<e^{\alpha_{n}} .
$$

It follows that

$$
\forall m \in \mathbb{N} \exists n_{m} \in \mathbb{N} \forall n \geq n_{m}: m \log n<\alpha_{n} .
$$

This implies that necessarily $\alpha_{n}>0$ for all $n \geq n_{m}$ and so

$$
\forall m \in \mathbb{N} \exists n_{m} \in \mathbb{N} \forall n \geq n_{m}: \frac{\log n}{\alpha_{n}}<\frac{1}{m} .
$$

This means precisely that $\lim _{n \rightarrow \infty} \frac{\log n}{\alpha_{n}}=0$.

(ii) Fix $m \in \mathbb{N}$. Then there is $n_{0} \in \mathbb{N}$ with $n_{0} \geq N$ such that $\frac{\log n}{\alpha_{n}}<\frac{1}{m+1}$ for all $n \geq n_{0}$. Since $w(N)<1$ implies that $\alpha_{n}=-\log w(n)>0$ for all $n \geq n_{0}$, we can conclude that $(m+1) \log n<\alpha_{n}$, i.e., $n^{m+1} w(n)<1$ for all $n \geq n_{0}$. So, $\sup _{n \in \mathbb{N}} n^{m+1} w(n)<\infty$. It follows that

$$
n^{m} w(n) \leq \frac{1}{n} \sup _{r \in \mathbb{N}} r^{m+1} w(r), \quad n \in \mathbb{N},
$$

with $\frac{1}{n} \sup _{r \in \mathbb{N}} r^{m+1} w(r) \rightarrow 0$ as $n \rightarrow \infty$. By (3) $\Leftrightarrow(4)$ in Proposition 3.4(i) it follows that $w \in s$.

Remark 2. (i) Concerning condition (5) in Proposition 3.4 (for any given $1<p<\infty)$, we claim that the entire set $\Sigma \subseteq \sigma_{p t}\left(\mathrm{C}^{(p, w)}\right)$ whenever $\sigma_{p t}\left(C^{(p, w)}\right)$ is an infinite set. To see this, suppose that $\frac{1}{m} \in \sigma_{p t}\left(C^{(p, w)}\right)$ for some $m \in \mathbb{N}$. According to the argument in Step 7 of the proof of Theorem 3.3, we can conclude that $\left(n^{m-1}\right)_{n \in \mathbb{N}} \in \ell_{p}(w)$. So, for all $1 \leq k<m$, it follows that

$$
\sum_{n=1}^{\infty}\left(n^{k}\right)^{p} w(n) \leq \sum_{n=1}^{\infty}\left(n^{m-1}\right)^{p} w(n)<\infty
$$


and hence, via (9), that the vector $\left(x_{n}\right)_{n \in \mathbb{N}} \in \mathbb{C}^{\mathbb{N}}$ given by (26), with $k$ in place of $m$, also belongs to $\ell_{p}(w)$, i.e., it is an eigenvector of $\mathrm{C}^{(p, w)}$ corresponding to $\lambda=\frac{1}{k}$. This shows that $\left\{\frac{1}{k}\right\}_{k=1}^{m} \subseteq \sigma_{p t}\left(\mathrm{C}^{(p, w)}\right)$ whenever $\frac{1}{m} \in \sigma_{p t}\left(\mathrm{C}^{(p, w)}\right)$, which clearly implies the stated claim.

(ii) Let $1<p_{0}<\infty$. The constant vector $\mathbf{1}:=(1,1, \ldots) \in \mathbb{C}^{\mathbb{N}}$ satisfies $\mathbf{C} \mathbf{1}=\mathbf{1}$ and so $1 \in \sigma_{p t}\left(C^{\left(p_{0}, w\right)}\right)$ if and only if, $\mathbf{1} \in \ell_{p_{0}}(w)$, i.e., if, and only if, $w \in \ell_{1}$. In this case, $1 \in \sigma_{p t}\left(C^{(p, w)}\right)$ for every $1<p<\infty$. Then Theorem 3.3(ii) implies that necessarily $t_{0} \in(0, \infty]$.

(iii) Let $w(n)=\frac{1}{n^{\alpha}}$, for all $n \in \mathbb{N}$ and some $\alpha>0$. Then $\sum_{n=1}^{\infty} n^{t} w(n)<$ $\infty$ if, and only if, $t<(\alpha-1)$ and so $t_{0}=(\alpha-1)$. In particular, $R_{w} \neq \mathbb{R}$. Moreover, for any $1<p<\infty$, we have

$$
\left\{\frac{1}{m}: m \in \mathbb{N}, 1 \leq m<\frac{t_{0}}{p}+1\right\}=\left\{\frac{1}{m}: m \in \mathbb{N}, 1 \leq m<\frac{(\alpha-1)}{p}+1\right\} .
$$

So, given any $1<p<\infty$, it is possible to choose an appropriate $\alpha>0$ such that $\sigma_{p t}\left(\mathrm{C}^{(p, w)}\right)$ is a finite set with any pre-assigned cardinality; see (16).

(iv) Condition (1) of Proposition 3.4, i.e., $R_{w}=\mathbb{R}$, implies that necessarily $S_{w}(p)=\emptyset$ for every $1<p<\infty$; see Proposition 3.1(i).

Let $w=(w(n))_{n \in \mathbb{N}}$ be any decreasing, (strictly) positive sequence and let $1<p<\infty$. The Cesàro operator $\mathrm{C}^{(p, w)}$ is similar (via an isometry) to an operator $T_{w} \in \mathcal{L}\left(\ell_{p}\right)$ which is defined by the factorable matrix $A(w)=$ $\left(a_{n k}\right)_{n, k \in \mathbb{N}}$ with entries $a_{n k}=a_{n} b_{k}=\frac{w(n)^{1 / p}}{n} \cdot w(k)^{-1 / p}$ for $1 \leq k \leq n$ and $a_{n k}=0$ for $k>n$ (see the proof of Lemma 2.1). In particular, $\sigma\left(\mathrm{C}^{(p, w)}\right)=$ $\sigma\left(T_{w}\right)$. Moreover, the matrix $A(w)$ satisfies the following two conditions:

(i) $\sup _{n \in \mathbb{N}} \sum_{k=1}^{\infty}\left|a_{n k}\right|=\sup _{n \in \mathbb{N}} \frac{w(n)^{1 / p}}{n} \sum_{k=1}^{n} w(k)^{-1 / p} \leq 1$, because $w$ decreasing implies that $\sum_{k=1}^{n} w(k)^{-1 / p} \leq n w(n)^{-1 / p}, n \in \mathbb{N}$, and

(ii) $f_{k}:=\lim _{n \rightarrow \infty} a_{n k}=w(k)^{-1 / p} \lim _{n \rightarrow \infty} \frac{w(n)^{1 / p}}{n}=0, k \in \mathbb{N}$, because $w \in \ell_{\infty}$.

If, in addition, the matrix $A(w)$ also satisfies the condition

(iii) $\alpha:=\lim _{n \rightarrow \infty} \sum_{k=1}^{\infty} a_{n k}=\lim _{n \rightarrow \infty} \frac{w(n)^{1 / p}}{n} \sum_{k=1}^{n} w(k)^{-1 / p}$ exists,

then the linear operator corresponding to $A(w)$ is a selfmap of $c$, the space of all convergent sequences, that is, $A(w)$ is conservative, [20, p.112].

Suppose now that the matrix $A(w)$ satisfies condition (iii) with $\alpha=1$. Then $A(w)$ is regular and the linear operator corresponding to $A(w)$ is limit preserving over $c$, [20, p.114]. Define $\eta:=\lim _{\sup _{n \rightarrow \infty}} a_{n} b_{n}$. For the operator $T_{w}$ (which is similar to the Cesàro operator $\mathrm{C}^{(p, w)}$ ) it turns out that $\eta=0$ and so a result of Rhoades and Yildirim [20, Theorem 3] yields that

$$
\left\{\lambda \in \mathbb{C}:\left|\lambda-\frac{1}{2}\right| \leq \frac{1}{2}\right\} \subseteq \sigma\left(\mathbb{C}^{(p, w)}\right),
$$


after noting that $S:=\overline{\left\{a_{n} b_{n}: n \in \mathbb{N}\right\}}=\Sigma_{0} \subseteq\left\{\lambda \in \mathbb{C}:\left|\lambda-\frac{1}{2}\right| \leq \frac{1}{2}\right\}$.

It is worthwhile to compare (14) with (30). So, let $1<p<\infty$ and $w$ be a positive, decreasing sequence such that $S_{w}(p) \neq \emptyset$. Then

$$
\left\{\lambda \in \mathbb{C}:\left|\lambda-\frac{1}{2}\right| \leq \frac{1}{2}\right\} \subseteq\left\{\lambda \in \mathbb{C}:\left|\lambda-\frac{p^{\prime}}{2 s_{p}}\right| \leq \frac{p^{\prime}}{2 s_{p}}\right\} \subseteq \sigma\left(\mathbb{C}^{(p, w)}\right)
$$

with the first inclusion holding if, and only if, $s_{p} \leq p^{\prime}$. Observe that if $\left(\frac{w(n)^{-1 / p}}{n}\right)_{n \in \mathbb{N}} \in \ell_{p^{\prime}}$, then $s_{p} \leq p^{\prime}$ is valid and conversely, if $s_{p}<p^{\prime}$, then $\left(\frac{w(n)^{-1 / p}}{n}\right)_{n \in \mathbb{N}} \in \ell_{p^{\prime}}$. In this case, (14) is a better inclusion than (30). For instance, if $w(n):=\frac{1}{n^{r}}$ for all $n \in \mathbb{N}$ and some $r>0$, then $\left(\frac{w(n)^{-1 / p}}{n}\right)_{n \in \mathbb{N}} \in$ $\ell_{p^{\prime}}$ if, and only if, $r<1$. On the other hand, the reverse inclusion

$$
\left\{\lambda \in \mathbb{C}:\left|\lambda-\frac{p^{\prime}}{2 s_{p}}\right| \leq \frac{p^{\prime}}{2 s_{p}}\right\} \subseteq\left\{\lambda \in \mathbb{C}:\left|\lambda-\frac{1}{2}\right| \leq \frac{1}{2}\right\}
$$

holds if, and only if, $p^{\prime} \leq s_{p}$. Observe that if $\left(\frac{w(n)^{-1 / p}}{n}\right)_{n \in \mathbb{N}} \notin \ell_{p^{\prime}}$, then $p^{\prime} \leq s_{p}$ is valid and conversely, if $p^{\prime}<s_{p}$, then $\left(\frac{w(n)^{-1 / p}}{n}\right)_{n \in \mathbb{N}} \notin \ell_{p^{\prime}}$. In this case, modulo the additional requirement that $\alpha=1$ (see condition (iii)), in which case (30) is actually valid, we see that (30) is a better inclusion than (14).

The following example shows that condition (iii) above and the property $S_{w}(p) \neq \emptyset$ can be compatible.

EXAmple 4. Fix $1<p<\infty$. For each $n \in \mathbb{N}$ set $w(n)=\frac{1}{(\log (n+1))^{p}}$, in which case $w(n) \downarrow 0$. Then $S_{w}(p)=(1, \infty)$ and

$$
\left\{\lambda \in \mathbb{C}:\left|\lambda-\frac{p^{\prime}}{2}\right| \leq \frac{p^{\prime}}{2}\right\}=\sigma\left(C^{(p, w)}\right) \text { with } \sigma_{p t}\left(C^{(p, w)}\right)=\emptyset ;
$$

see Example 3(i) with $\gamma=p$. Moreover, concerning condition (iii) observe that

$$
\frac{w(n)^{1 / p}}{n} \sum_{k=1}^{n} w(k)^{-1 / p}=\frac{1}{n \log (n+1)} \sum_{k=1}^{n} \log (k+1), \quad n \in \mathbb{N} .
$$

The inequalities

$[(n+1) \log (n+1)-n] \leq \sum_{k=1}^{n} \log (k+1) \leq[(n+2) \log (n+2)-n-2 \log 2], \quad n \in \mathbb{N}$,

then imply that

$$
\alpha=\lim _{n \rightarrow \infty} \frac{w(n)^{1 / p}}{n} \sum_{k=1}^{n} w(k)^{-1 / p}=1 .
$$

Note also that $\left(\frac{w(n)^{-1 / p}}{n}\right)_{n \in \mathbb{N}}=\left(\frac{\log (n+1)}{n}\right)_{n \in \mathbb{N}} \in \ell_{p^{\prime}}$. 
We conclude this section with some comments about the mean ergodicity and the linear dynamics of $\mathrm{C}^{(p, w)}$. For $X$ a Banach space, recall that $T \in$ $\mathcal{L}(X)$ is mean ergodic if its sequence of Cesàro averages $T_{[n]}:=\frac{1}{n} \sum_{m=1}^{n} T^{m}$, for $n \in \mathbb{N}$, converges to some operator $P \in \mathcal{L}(X)$ for the strong operator topology, i.e., $\lim _{n \rightarrow \infty} T_{[n]} x=P x$ for each $x \in X$, [10, Ch.VIII]. Since $\frac{1}{n} T^{n}=T_{[n]}-\frac{n-1}{n} T_{[n-1]}$, for $n \in \mathbb{N}$ (with $T_{[0]}:=I$ ), a necessary condition for $T$ to be mean ergodic is that $\lim _{n \rightarrow \infty} \frac{1}{n} T^{n}=0$ (in the strong operator topology).

Let $w$ be a positive, decreasing sequence and $1<p<\infty$ with $S_{p}(w) \neq \emptyset$. If $s_{p}<p^{\prime}$, then it follows from (12) that $\mu:=\frac{1}{2}\left(1+\frac{p^{\prime}}{s_{p}}\right) \in \sigma_{p t}\left(\left(\mathrm{C}^{(p, w)}\right)^{\prime}\right)$ and so there exists a non-zero vector $x^{\prime} \in \ell_{p^{\prime}}\left(w^{-p^{\prime} / p}\right)$ such that $\left(\mathrm{C}^{(p, w)}\right)^{\prime} x^{\prime}=\mu x^{\prime}$. Choose any $x \in \ell_{p}(w) \backslash\{0\}$ satisfying $\left\langle x, x^{\prime}\right\rangle \neq 0$. Then

$$
\left\langle\frac{1}{n}\left(\mathrm{C}^{(p, w)}\right)^{n} x, x^{\prime}\right\rangle=\frac{1}{n}\left\langle x,\left(\left(\mathrm{C}^{(p, w)}\right)^{\prime}\right)^{n} x^{\prime}\right\rangle=\frac{\mu^{n}}{n}\left\langle x, x^{\prime}\right\rangle, \quad n \in \mathbb{N},
$$

with $\mu>1$ and so the set $\left\{\frac{1}{n}\left(\mathrm{C}^{(p, w)}\right)^{n} x: n \in \mathbb{N}\right\}$ is unbounded in $\ell_{p}(w)$. In particular, the sequence $\left\{\frac{1}{n}\left(\mathrm{C}^{(p, w)}\right)^{n}\right\}_{n \in \mathbb{N}}$ cannot converge to 0 for the strong operator topology in $\mathcal{L}\left(\ell_{p}(w)\right)$. Accordingly, $\mathrm{C}^{(p, w)}$ fails to be mean ergodic whenever $s_{p}<p^{\prime}$. This is the case when $w(n)=1$, for all $n \in \mathbb{N}$, in which case $s_{p}=1$, and we recover the known fact that the classical Cesàro operator $\mathrm{C}^{(p)}$ fails to be mean ergodic for every $1<p<\infty$; see [3, Section 4], where it is also shown that the Cesàro operator fails to be mean ergodic in the classical Banach sequence spaces $c_{0}, c, \ell_{p}(1<p \leq \infty), b v_{0}$ and $b v$ but, that it is mean ergodic in $b v_{p}(1<p<\infty)$. For $w$ as in Example 3(i) we recall that also $s_{p}=1$, for every $1<p<\infty$, and so $\mathrm{C}^{(p, w)}$ is not mean ergodic.

Concerning the dynamics of a continuous linear operator $T$ defined on a separable Banach space $X$, recall that $T$ is hypercyclic if there exists $x \in X$ such that the orbit $\left\{T^{n} x: n \in \mathbb{N}_{0}\right\}$ is dense in $X$. If, for some $x \in X$, the projective orbit $\left\{\lambda T^{n} x: \lambda \in \mathbb{C}, n \in \mathbb{N}_{0}\right\}$ is dense in $X$, then $X$ is called supercyclic. Clearly, hypercyclicity always implies supercyclicity.

Let now $w$ be a positive, decreasing sequence and $1<p<\infty$. According to Remark 1(iv) the infinite set $\Sigma \subseteq \sigma_{p t}\left(\left(\mathrm{C}^{(p, w)}\right)^{\prime}\right)$. Then, by a result of Ansari and Bourdon [4, Theorem 3.2], $\mathrm{C}^{(p, w)}$ is not supercyclic and hence, also not hypercyclic.

\section{Compactness of $C^{(p, w)}$}

According to (27), for each $1<p<\infty$ the classical Cesàro operator $\mathrm{C}^{(p)} \in \mathcal{L}\left(\ell_{p}\right)$ is surely not compact. However, in the presence of a positive weight $w \downarrow 0$, this may no longer be the case for $\mathrm{C}^{(p, w)}$ acting on $\ell_{p}(w)$. We begin with the following fact.

Proposition 4.1. Let $w$ be a positive, decreasing weight. 
(i) For every $1<p<\infty$ we have $\Sigma \subseteq \sigma\left(\mathrm{C}^{(p, w)}\right)$.

(ii) Suppose that $\mathrm{C}^{(p, w)}$ is a compact operator, for some $1<p<\infty$. Then

$$
\sigma\left(\mathrm{C}^{(p, w)}\right)=\Sigma_{0} \text { and } \sigma_{p t}\left(\mathrm{C}^{(p, w)}\right)=\Sigma .
$$

Moreover, $w \in s$ and $r\left(\mathrm{C}^{(p, w)}\right)<\left\|\mathrm{C}^{(p, w)}\right\|$.

Proof. (i) According to Remark 1(iv) we have $\Sigma \subseteq \sigma_{p t}\left(\left(\mathrm{C}^{(p, w)}\right)^{\prime}\right)$. But, always $\sigma_{p t}\left(\left(\mathrm{C}^{(p, w)}\right)^{\prime}\right) \subseteq \sigma\left(\mathrm{C}^{(p, w)}\right),\left[10\right.$, p. 581], and so $\Sigma \subseteq \sigma\left(\mathrm{C}^{(p, w)}\right)$.

(ii) Since $\mathrm{C}^{(p, w)}$ is injective, $0 \notin \sigma_{p t}\left(\mathrm{C}^{(p, w)}\right)$. The compactness of $\mathrm{C}^{(p, w)}$ then implies that $\sigma_{p t}\left(\mathrm{C}^{(p, w)}\right)=\sigma\left(\mathrm{C}^{(p, w)}\right) \backslash\{0\}$, [16, Theorem 3.4.23]. According to the proof of Step 8 for Theorem 3.3 we also have that $\sigma_{p t}\left(\mathrm{C}^{(p, w)}\right) \subseteq \Sigma$. In view of part (i), the equalities in (31) follow.

By Theorem 3.3(ii) we must have $R_{w}=\mathbb{R}$ (if not, then $t_{0}$ is finite and so (16) would imply that $\sigma_{p t}\left(\mathrm{C}^{(p, w)}\right)$ is finite which is a contradiction to (31). Then, via Proposition 3.4(i), we can conclude that $w \in s$.

It follows from (4) and the equality $r\left(\mathrm{C}^{(p, w)}\right)=1$ (see (31)) that $r\left(\mathrm{C}^{(p, w)}\right)<$ $\left\|\mathrm{C}^{(p, w)}\right\|$.

To decide when $C^{(p, w)}$ is compact, first observe that $C^{(p, w)}=\Phi_{w}^{-1} T_{w} \Phi_{w}$ (see Lemma 2.1 and its proof), where $T_{w} \in \mathcal{L}\left(\ell_{p}\right)$ is given by (3). Given any $x \in B_{p}:=\left\{x \in \ell_{p}:\|x\| \leq 1\right\}$ and $i \in \mathbb{N}$, it follows from Hölder's inequality that

$$
\begin{aligned}
\sum_{n=i}^{\infty}\left|\left(T_{w} x\right)_{n}\right|^{p} & =\sum_{n=i}^{\infty} \frac{w(n)}{n^{p}}\left|\sum_{k=1}^{n} \frac{1}{w(k)^{1 / p}} \cdot x_{k}\right|^{p} \\
& \leq \sum_{n=i}^{\infty} \frac{w(n)}{n^{p}}\left(\sum_{k=1}^{n} \frac{1}{w(k)^{p^{\prime} / p}}\right)^{p / p^{\prime}} .
\end{aligned}
$$

So, $T_{w}$ (hence, also $\mathrm{C}^{(p, w)}$ ) will be compact whenever $w$ satisfies the following

$$
\text { Compactness criterion: } \quad \sum_{n=1}^{\infty} \frac{w(n)}{n^{p}}\left(\sum_{k=1}^{n} \frac{1}{w(k)^{p^{\prime} / p}}\right)^{p / p^{\prime}}<\infty .
$$

Indeed, (32) implies that $\lim _{i \rightarrow \infty} \sum_{n=i}^{\infty}\left|\left(T_{w} x\right)_{n}\right|^{p}=0$ uniformly with respect to $x \in B_{p}$, from which the relative compactness in $\ell_{p}$ of the bounded set $T_{w}\left(B_{p}\right) \subseteq \ell_{p}$ follows, [10, pp.338-339].

We introduce some notation. Let $w$ be a positive, decreasing sequence. Define

$$
A_{n}(p, w):=w(n)^{p^{\prime} / p} \sum_{k=1}^{n} \frac{1}{w(k)^{p^{\prime} / p}}, \quad n \in \mathbb{N}, 1<p<\infty .
$$

The compactness criterion (32) then states that $\mathrm{C}^{(p, w)}$ is a compact operator if $\sum_{n=1}^{\infty}\left(A_{n}(p, w)\right)^{p / p^{\prime}} / n^{p}<\infty$. 
TheOREm 4.2. Suppose, for some $1<p<\infty$, that there exist constants $M>0$ and $0 \leq \alpha<1$ such that

$$
A_{n}(p, w) \leq M n^{\alpha}, \quad n \in \mathbb{N} .
$$

Then $\mathrm{C}^{(q, w)}$ is a compact operator for every $1<q \leq p$. In particular, $w \in s$.

Proof. Observe, for fixed $1<q \leq p$, that

$$
\gamma:=\frac{q^{\prime}}{q}-\frac{p^{\prime}}{p}=\frac{1}{q-1}-\frac{1}{p-1}=\frac{p-q}{(q-1)(p-1)} \geq 0 .
$$

For each $n \in \mathbb{N}$ we have

$$
\sum_{k=1}^{n} \frac{1}{w(k)^{q^{\prime} / q}}=\sum_{k=1}^{n} \frac{1}{w(k)^{p^{\prime} / p}} \cdot w(k)^{-\gamma} .
$$

Accordingly, for each $n \in \mathbb{N}$,

$$
\begin{aligned}
A_{n}(q, w) & =\frac{w(n)^{q^{\prime} / q}}{w(n)^{p^{\prime} / p}} \cdot w(n)^{p^{\prime} / p} \sum_{k=1}^{n} \frac{1}{w(k)^{p^{\prime} / p}} \cdot w(k)^{-\gamma} \\
& =w(n)^{p^{\prime} / p} \sum_{k=1}^{n} \frac{1}{w(k)^{p^{\prime} / p}} \cdot\left(\frac{w(n)}{w(k)}\right)^{\gamma} .
\end{aligned}
$$

Since $w$ is decreasing, $\frac{w(n)}{w(k)} \leq 1$ for all $1 \leq k \leq n$ and so

$$
A_{n}(q, w) \leq w(n)^{p^{\prime} / p} \sum_{k=1}^{n} \frac{1}{w(k)^{p^{\prime} / p}}=A_{n}(p, w) \leq M n^{\alpha} .
$$

Accordingly,

$$
\sum_{n=1}^{\infty} \frac{\left(A_{n}(q, w)\right)^{q / q^{\prime}}}{n^{q}} \leq M^{q / q^{\prime}} \sum_{n=1}^{\infty} \frac{n^{\alpha q / q^{\prime}}}{n^{q}}=M^{q / q^{\prime}} \sum_{n=1}^{\infty} \frac{1}{n^{q-\left(\alpha q / q^{\prime}\right)}} .
$$

But, $q-\frac{\alpha q}{q^{\prime}}=q-\alpha(q-1)=q(1-\alpha)+\alpha>(1-\alpha)+\alpha=1$ and so

$$
\sum_{n=1}^{\infty} \frac{\left(A_{n}(q, w)\right)^{q / q^{\prime}}}{n^{q}}<\infty
$$

Then the compactness criterion yields that $C^{(q, w)}$ is a compact operator.

That $w \in s$ is a consequence of Proposition 4.1(ii).

The following consequence of Theorem 4.2 leads to a rich supply of weights $w$ for which $\mathrm{C}^{(p, w)}$ is compact. 
COROLlary 4.3. Let $w$ be a positive weight with $w \downarrow 0$. If the limit

$$
l:=\lim _{n \rightarrow \infty} \frac{w(n)}{w(n-1)}
$$

exists in $\mathbb{R} \backslash\{1\}$, then $\mathrm{C}^{(p, w)}$ is compact for every $1<p<\infty$.

Proof. Fix $1<p<\infty$. According to Theorem 4.2 (with $\alpha=0$ ) it suffices to prove that $\sup _{n \in \mathbb{N}} A_{n}(p, w)<\infty$. Set $a_{n}:=\sum_{k=1}^{n} w(k)^{-p^{\prime} / p}$ and $b_{n}:=w(n)^{-p^{\prime} / p}$ for $n \in \mathbb{N}$. Since $w \downarrow 0$, we have $b_{n} \uparrow \infty$. Moreover, the limit

$$
\begin{aligned}
& \lim _{n \rightarrow \infty} \frac{a_{n}-a_{n-1}}{b_{n}-b_{n-1}}=\lim _{n \rightarrow \infty} \frac{w(n)^{-p^{\prime} / p}}{w(n)^{-p^{\prime} / p}-w(n-1)^{-p^{\prime} / p}} \\
& =\lim _{n \rightarrow \infty} \frac{1}{1-(w(n) / w(n-1))^{p^{\prime} / p}}=\frac{1}{1-l^{p^{\prime} / p}}
\end{aligned}
$$

exists in $\mathbb{R}$ as $l \neq 1$. According to the Stolz-Cesàro criterion, [17, Theorem 1.22], it follows that $\lim _{n \rightarrow \infty} \frac{a_{n}}{b_{n}}=1 /\left(1-l^{p^{\prime} / p}\right) \in \mathbb{R}$, i.e., $\lim _{n \rightarrow \infty} A_{n}(p, w)=$ $1 /\left(1-l^{p^{\prime} / p}\right) \in \mathbb{R}$. In particular, $\sup _{n \in \mathbb{N}} A_{n}(p, w)<\infty$ is indeed satisfied.

REMARK 3. (i) Let $w$ be a positive, decreasing weight.

(a) According to (14), if $\mathrm{C}^{(p, w)}$ is a compact operator for some $1<p<\infty$, then $S_{w}(p)=\emptyset$.

(b) The condition $w \downarrow 0$ by itself need not imply that $S_{w}(p)=\emptyset$ (see Examples 3, for instance).

(ii) Suppose $S_{w}(p) \neq \emptyset$ for some $1<p<\infty$. Then $\mathrm{C}^{(q, w)}$ fails to be compact for every $q \in[p, \infty)$. This follows from part (i)(a) and Proposition 3.1(iii).

(iii) The following examples (a)-(c) all fall within the scope of Corollary 4.3. So, in each case $w \in s$ and the identities in (31) hold; see Proposition 4.1 .

(a) For any fixed $a>1$ and $r \geq 0$ set $w(n):=n^{r} / a^{n}$ for $n \in \mathbb{N}$. Then

$$
\lim _{n \rightarrow \infty} \frac{w(n)}{w(n-1)}=a^{-1} \neq 1
$$

(b) For any fixed $a \geq 1$, the weight $w(n):=a^{n} / n$ ! for $n \in \mathbb{N}$ satisfies

$$
\lim _{n \rightarrow \infty} \frac{w(n)}{w(n-1)}=0 \neq 1
$$

(c) The weight $w(n):=1 / n^{n}$ for $n \in \mathbb{N}$ also satisfies (35). 
We point out, since $w$ is decreasing, that $\frac{w(n)}{w(n-1)} \in(0,1]$ for all $n \in \mathbb{N}$. Hence, whenever the limit (34) exists, then necessarily $l \in[0,1]$.

As an application, suppose that the positive, decreasing weight $w$ has the property that $l:=\lim _{n \rightarrow \infty} \frac{w(n)}{w(n-1)}$ exists in $[0,1)$. Then, for each $r>$ 0 , the positive, decreasing weight $w^{r}: n \mapsto w(n)^{r}$, for $n \in \mathbb{N}$, satisfies $\lim _{n \rightarrow \infty} \frac{w(n)^{r}}{w(n-1)^{r}}=l^{r} \in[0,1)$. Hence, $\mathrm{C}^{\left(p, w^{r}\right)}$ is a compact operator for every $1<p<\infty$.

(iv) The following criterion is sufficient to ensure that the limit (34) exists in $\mathbb{R} \backslash\{1\}$. Hence, both Proposition 4.1 and Corollary 4.3 are applicable to such a weight $w$. In particular, $w \in s$.

Let $\beta=\left(\beta_{n}\right)_{n \in \mathbb{N}}$ be a positive, increasing sequence with $\beta \uparrow \infty$ such that $\lim _{n \rightarrow \infty}\left(\beta_{n}-\beta_{n-1}\right)=\infty$. Then the weight $w(n):=e^{-\beta_{n}}$, for $n \in \mathbb{N}$, satisfies $l:=\lim _{n \rightarrow \infty} \frac{w(n)}{w(n-1)}=0 \neq 1$.

It is routine to verify that $\lim _{n \rightarrow \infty} \frac{w(n)}{w(n-1)}=0$.

For the weight $w(n):=a^{-n}$ for $n \in \mathbb{N}$ (with $a>1$ ) we have that $\beta_{n}:=-\log w(n)=n \log (a) \uparrow \infty$ but, $\left(\beta_{n}-\beta_{n-1}\right) \log (a) \not \rightarrow \infty$ for $n \rightarrow \infty$. So, the above criterion is not applicable to this weight. However, according to part (iii)(a) of this remark (with $r=0$ ) the weight $w$ is admissible for Corollary 4.3 .

The following examples illustrate that Theorem 4.2 is more general than Corollary 4.3.

Example 5. (i) Fix $0<\beta<1$ and set $w_{\beta}(n):=e^{-n^{\beta}}$ for $n \in \mathbb{N}$, in which case $w \downarrow 0$, but

$$
\lim _{n \rightarrow \infty} \frac{w_{\beta}(n)}{w_{\beta}(n-1)}=\lim _{n \rightarrow \infty} e^{(n-1)^{\beta}-n^{\beta}}=\lim _{n \rightarrow \infty} e^{-\beta / n^{(1-\beta)}}=1,
$$

as $(n-1)^{\beta}-n^{\beta}=n^{\beta}\left[\left(1-\frac{1}{n}\right)^{\beta}-1\right]=n^{\beta}\left[1-\frac{\beta}{n}+o\left(\frac{1}{n}\right)-1\right] \simeq-\frac{\beta}{n^{1-\beta}}$ for $n \rightarrow \infty$. So, Corollary 4.3 is not applicable. We show that Theorem 4.2 does apply.

Fix $1<p<\infty$ and set $\gamma:=\frac{p^{\prime}}{p}$. Then, for each $n \in \mathbb{N}$, we have that

$$
\begin{aligned}
A_{n}\left(p, w_{\beta}\right) & =e^{-\gamma n^{\beta}} \sum_{k=1}^{n} e^{\gamma k^{\beta}} \leq e^{-\gamma n^{\beta}} \int_{1}^{n+1} e^{\gamma x^{\beta}} d x \\
& =\frac{e^{-\gamma n^{\beta}}}{\beta} \int_{1}^{(n+1)^{\beta}} e^{\gamma t} t^{\frac{1}{\beta}-1} d t \leq \frac{e^{-\gamma n^{\beta}}}{\beta} \int_{1}^{(n+1)^{\beta}} e^{\gamma t} t^{m} d t,
\end{aligned}
$$

where $m \in \mathbb{N}$ is chosen minimal such that $(m-1)<\frac{1}{\beta}-1 \leq m$. An integration by parts $(m+1)$-times yields that

$$
\begin{aligned}
\int_{1}^{(n+1)^{\beta}} e^{\gamma t} t^{m} d t & \leq a_{0}+a_{1}(n+1)^{\beta} e^{\gamma(n+1)^{\beta}}+a_{2}(n+1)^{2 \beta} e^{\gamma(n+1)^{\beta}} \\
& +\ldots+a_{m}(n+1)^{m \beta} e^{\gamma(n+1)^{\beta}}
\end{aligned}
$$


for positive constants $a_{0}, a_{1}, \ldots, a_{m}$. It follows that

$$
\int_{1}^{(n+1)^{\beta}} e^{\gamma t} t^{m} d t \leq M(1+n)^{m \beta} e^{\gamma(1+n)^{\beta}}, \quad n \in \mathbb{N},
$$

for some constant $M>0$. Accordingly,

$$
A_{n}\left(p, w_{\beta}\right) \leq \frac{M}{\beta}(1+n)^{m \beta} e^{\gamma\left((1+n)^{\beta}-n^{\beta}\right)}, \quad n \in \mathbb{N} .
$$

Since $(n+1)^{\beta}-n^{\beta} \simeq \frac{\beta}{n^{1-\beta}}$ and $(1+n)^{m \beta} \simeq n^{m \beta}$ for $n \rightarrow \infty$, there exists $K>0$ (independent of $n$ ) such that

$$
A_{n}\left(p, w_{\beta}\right) \leq K n^{m \beta}, \quad i \in \mathbb{N} .
$$

Since $(m-1)<\frac{1}{\beta}-1$ implies that $\alpha:=m \beta \in(0,1)$, Theorem 4.2 yields that $\mathrm{C}^{\left(p, w_{\beta}\right)}$ is compact.

For $\beta \geq 1$ the compactness of $\mathrm{C}^{\left(p, w_{\beta}\right)}$ follows from Corollary 4.3. Indeed, if $\beta=1$, then $w_{\beta}(n)=e^{-n}$ for $n \in \mathbb{N}$ and so Remark 3(iii)(a) implies the compactness of $\mathrm{C}^{\left(p, w_{\beta}\right)}$. For $\beta>1$, observe from above that

$$
\lim _{n \rightarrow \infty} \frac{w_{\beta}(n)}{w_{\beta}(n-1)}=\lim _{n \rightarrow \infty} e^{(n-1)^{\beta}-n^{\beta}}=\lim _{n \rightarrow \infty} e^{-\beta n^{\beta-1}}=0
$$

and so the compactness of $\mathrm{C}^{\left(p, w_{\beta}\right)}$ follows again from Corollary 4.3.

(ii) There also exist positive, decreasing weights $w \in s$ such that the sequence $\left\{\frac{w(n)}{w(n-1)}\right\}_{n \in \mathbb{N}}$ fails to converge at all, yet $\mathrm{C}^{(p, w)}$ is a compact operator for every $1<p<\infty$.

Define $w(n):=\frac{1}{j^{j}}, n=2 j-1$, and $w(n):=\frac{1}{2 j^{j}}, n=2 j$, for each $j \in \mathbb{N}$. Then $w$ is (strictly) decreasing to 0 . For $n_{j}:=2 j, j \in \mathbb{N}$, we have $\frac{w\left(n_{j}\right)}{w\left(n_{j}-1\right)}=\frac{1}{2}$ for all $j \in \mathbb{N}$ and so $\lim _{j \rightarrow \infty} \frac{w\left(n_{j}\right)}{w\left(n_{j}-1\right)}=\frac{1}{2}$, whereas for $n_{r}:=$ $2 r+1, r \in \mathbb{N}$, the subsequence $\left\{\frac{w\left(n_{r}\right)}{w\left(n_{r}-1\right)}\right\}_{r \in \mathbb{N}}$ of $\left\{\frac{w(n)}{w(n-1)}\right\}_{n \in \mathbb{N}}$ converges to 0 . Accordingly, the sequence $\left\{\frac{w(n)}{w(n-1)}\right\}_{n \in \mathbb{N}}$ is not convergent and so Corollary 4.3 is not applicable.

Fix $1<p<\infty$ and set $\gamma:=\frac{p^{\prime}}{p}>0$. To establish the compactness of $\mathrm{C}^{(p, w)}$ observe, for every $j \in \mathbb{N}$, that

$$
A_{2 j}(p, w)=\frac{1}{\left(2 j^{j}\right)^{\gamma}}\left(\sum_{k=1}^{j}\left(k^{k}\right)^{\gamma}+\sum_{k=1}^{j}\left(2 k^{k}\right)^{\gamma}\right)=\frac{1+2^{\gamma}}{2^{\gamma}} \frac{1}{\left(j^{j}\right)^{\gamma}} \sum_{k=1}^{j}\left(k^{k}\right)^{\gamma},
$$

and that

$$
A_{2 j-1}(p, w)=1+\frac{1}{\left(j^{j}\right)^{\gamma}} \sum_{k=1}^{2(j-1)} w(k)^{-\gamma}=1+\frac{(j-1)^{(j-1) \gamma}}{\left(j^{j}\right)^{\gamma}} A_{2(j-1)}(p, w),
$$


with $\lim _{j \rightarrow \infty} \frac{(j-1)^{(j-1) \gamma}}{\left(j^{j}\right)^{\gamma}}=0$. Set $a_{j}:=\sum_{k=1}^{j}\left(k^{k}\right)^{\gamma}$ and $b_{j}:=\left(j^{j}\right)^{\gamma}$ for $j \in \mathbb{N}$. Then $b_{j} \uparrow \infty$. Moreover,

$$
\lim _{j \rightarrow \infty} \frac{a_{j}-a_{j-1}}{b_{j}-b_{j-1}}=\lim _{j \rightarrow \infty} \frac{\left(j^{j}\right)^{\gamma}}{\left(j^{j}\right)^{\gamma}-\left((j-1)^{j-1}\right)^{\gamma}}=\lim _{j \rightarrow \infty} \frac{1}{1-\frac{(j-1)^{(j-1) \gamma}}{\left(j^{j}\right)^{\gamma}}}=1 .
$$

According to the Stolz-Cesàro criterion, [17, Theorem 1.22], it follows that also $\lim _{j \rightarrow \infty} \frac{a_{j}}{b_{j}}=1$. So, via (36) and (37) we obtain that $\lim _{j \rightarrow \infty} A_{2 j}(p, w)=$ $\frac{1+2^{\gamma}}{2^{\gamma}}$ and $\lim _{j \rightarrow \infty} A_{2 j-1}(p, w)=1$. In particular, $\sup _{i \in \mathbb{N}} A_{i}(p, w)<\infty$ and so Theorem 4.2 applies (with $\alpha=0$ ). Hence, $\mathrm{C}^{(p, w)}$ is compact and $w \in s$.

The following result is a comparison type criterion for compactness. One knows something about the compactness of $\mathrm{C}^{(p, w)}$ in $\ell_{p}(w)$ for a certain weight $w$ and $1<p<\infty$ and one has a second weight $v$ whose growth relative to $w$ is controlled. Then also $\mathrm{C}^{(p, v)} \in \mathcal{L}\left(\ell_{p}(v)\right)$ is compact.

Proposition 4.4. Let $w$ be a positive, decreasing sequence. Suppose, for some $1<p<\infty$, that there exists $0 \leq \alpha<1$ such that

$$
A_{n}(p, w) \leq M n^{\alpha}, \quad n \in \mathbb{N},
$$

for some constant $M>0$.

Let $v$ be any positive, decreasing sequence such that $\left\{\frac{v(n)}{w(n)}\right\}_{n \in \mathbb{N}} \in \ell_{\infty}$ and satisfying

$$
w(n) \leq K n^{\beta} v(n), \quad n \in \mathbb{N},
$$

for some $0 \leq \beta<(p-1)(1-\alpha)$ and some constant $K>0$. Then $\mathrm{C}^{(q, v)} \in$ $\mathcal{L}\left(\ell_{q}(v)\right)$ is a compact operator for every $1<q \leq p$.

Proof. Let $L:=\sup _{n \in \mathbb{N}} \frac{v(n)}{w(n)}$. Then, for each $n \in \mathbb{N}$, we have via (38) and (39) that

$$
\begin{aligned}
& A_{n}(p, v)=v(n)^{p^{\prime} / p} \sum_{k=1}^{n} \frac{1}{v(k)^{p^{\prime} / p}}=\left(\frac{v(n)}{w(n)}\right)^{p^{\prime} / p} w(n)^{p^{\prime} / p} \sum_{k=1}^{n} \frac{1}{w(k)^{p^{\prime} / p}} \cdot\left(\frac{w(k)}{v(k)}\right)^{p^{\prime} / p} \\
& \leq L^{p^{\prime} / p} w(n)^{p^{\prime} / p} \sum_{k=1}^{n} \frac{1}{w(k)^{p^{\prime} / p}}\left(K k^{\beta}\right)^{p^{\prime} / p} \leq(L K)^{p^{\prime} / p} w(n)^{p^{\prime} / p} \sum_{k=1}^{n} \frac{1}{w(k)^{p^{\prime} / p}} n^{\beta p^{\prime} / p} \\
& =(L K)^{p^{\prime} / p} n^{\beta p^{\prime} / p} A_{n}(p, w) \leq M(L K)^{p^{\prime} / p} n^{\alpha+\left(\beta p^{\prime} / p\right)} .
\end{aligned}
$$

Moreover, $\alpha+\frac{\beta p^{\prime}}{p}=\alpha+\frac{\beta}{(p-1)}<1$ because $0 \leq \beta<(p-1)(1-\alpha)$ implies $\frac{\beta}{(p-1)}<(1-\alpha)$ which implies $\alpha+\frac{\beta}{(p-1)}<1$. So, Theorem 4.2 applied to $v$ (with $\alpha+\frac{\beta}{(p-1)}$ in place of $\alpha$ ) implies that $\mathrm{C}^{(q, v)} \in \mathcal{L}\left(\ell_{q}(v)\right)$ is compact for all $1<q \leq p$. 
Example 6. Let $v(n):=\frac{1}{e^{n^{\beta} \log ^{\gamma}(n+1)}}$ for $n \in \mathbb{N}$, where $0<\beta<1$ and $\gamma>0$. Then $\mathrm{C}^{(p, v)} \in \mathcal{L}\left(\ell_{p}(v)\right)$ is compact for every $1<p<\infty$. Observe that $\lim _{n \rightarrow \infty} \frac{v(n)}{v(n-1)}=1$ and so Corollary 4.3 is not applicable.

So, fix $1<p<\infty$. Define $w(n):=e^{-n^{\beta}}$ for $n \in \mathbb{N}$. According to Example 5(i), there exist constants $M>0$ and $0<\alpha<1$ such that

$$
A_{n}(p, w) \leq M n^{\alpha}, \quad n \in \mathbb{N} .
$$

Since $v(n) \leq w(n)$ for $n \in \mathbb{N}$, it is clear that $\left\{\frac{v(n)}{w(n)}\right\}_{n \in \mathbb{N}} \in \ell_{\infty}$. Choose any $r \in(0,(p-1)(1-\alpha))$. Then

$$
\frac{w(n)}{v(n)}=\log ^{\gamma}(n+1)=\frac{\log ^{\gamma}(n+1)}{n^{r}} \cdot n^{r} \leq K n^{r}, \quad n \in \mathbb{N},
$$

for some $K>0$ (as $\left.\lim _{n \rightarrow \infty} \frac{\log ^{\gamma}(n+1)}{n^{r}}=0\right)$. According to Proposition 4.4, we can conclude that $C^{(p, v)}$ is compact in $\ell_{p}(v)$.

Acknowledgements. The research of the first two authors was partially supported by the projects MTM2013-43540-P and GVA Prometeo II/2013/013 (Spain).

\section{References}

[1] A.M. Akhmedov, F. Başar, On the fine spectrum of the Cesàro operator in $c_{0}$, Math. J. Ibaraki Univ. 36 (2004), 25-32.

[2] A.M. Akhmedov, F. Başar, The fine spectrum of the Cesàro operator $C_{1}$ over the sequence space $b v_{p},(1 \leq p<\infty)$, Math. J. Okayama Univ. 50 (2008), 135-147.

[3] A.A. Albanese, J. Bonet, W.J. Ricker, Convergence of arithmetic means of operators in Fréchet spaces, J. Math. Anal. Appl. 401 (2013), 160-173.

[4] S.I. Ansari, P.S. Bourdon, Some properties of cyclic operators, Acta Sci. Math. Szeged 63 (1997), 195-207.

[5] G. Bennett, Some elementary inequalities, Quart. J. Math. Oxford 38 (1987), 401-425.

[6] A. Brown, P.R. Halmos, A.L. Shields, Cesàro operators, Acta Sci. Math. Szeged 26 (1965), 125-137.

[7] G.P. Curbera, W.J. Ricker, Spectrum of the Cesàro operator in $\ell^{p}$, Arch. Math. 100 (2013), 267-271.

[8] G.P. Curbera, W.J. Ricker, Solid extensions of the Cesàro operator on $\ell^{p}$ and $c_{0}$, Integr. Equ. Oper. Theory 80 (2014), 61-77.

[9] G.P. Curbera, W.J. Ricker, The Cesàro operator and unconditional Taylor series in Hardy spaces, Integr. Equ. Oper. Theory, to appear. DOI 10.1007/s00020-015-2230-9. 
[10] N. Dunford, J.T. Schwartz, Linear Operators I: General Theory, 2nd Printing, Wiley Interscience Publ., New York, 1964.

[11] G.J.O. Jameson, Norms and lower bounds of operators on the Lorentz sequence space $d(w, 1)$, Illinois J. Math. 43 (1999), 79-99.

[12] G.J.O. Jameson, R. Lashkaripour, Norms of certain operators on weighted $\ell^{p}$ spaces and Lorentz sequences spaces, J. Inequal. Pure and Appl. Math. 3 Article 6, (2002), 17 pp.

[13] G.H. Hardy, Divergent series, Clarendon Press. Oxford, 1949.

[14] G.H. Hardy, J.E. Littlewood, G. Polya, Inequalities, Cambridge Univ. Press, Cambridge, 1964.

[15] G. Leibowitz, Spectra of discrete Cesàro operators, Tamkang J. Math. 3 (1972), 123-132.

[16] R.E. Megginson, An Introduction to Banach Space Theory, Springer, New York Berlin Heidelberg, 1998.

[17] M. Mureşan, A Concrete Approach to Classical Analysis, Springer, Berlin, 2008.

[18] J.I. Okutoyi, On the spectrum of $C_{1}$ as an operator on bvo, J. Austral. Math. Soc. (Series A) 48 (1990), 79-86.

[19] J.B. Reade, On the spectrum of the Cesàro operator, Bull. London Math. Soc. 17 (1985), 263-267.

[20] B.E. Rhoades, M. Yildirim, Spectra for factorable matrices on $\ell^{p}$, Integr. Equ. Oper. Theory 55 (2006), 111-126.

Dipartimento di Matematica e Fisica

"E. De Giorgi"

Università del Salento- C.P.193

I-73100 Lecce, Italy

angela.albanese@unisalento.it
Instituto Universitario de Matemática Pura y Aplicada

IUMPA

Universitat Politècnica de València E-46071 Valencia, Spain jbonet@mat.upv.es

Math.-Geogr. Fakultät

Katholische Universität Eichstätt-Ingolstadt

D-85072 Eichstätt, Germany

werner.ricker@ku-eichstaett.de 